\title{
The Mean-Field Limit for the Dynamics of Large Particle Systems
}

\author{
François Golse
}

\begin{abstract}
This short course explains how the usual mean-field evolution PDEs in Statistical Physics — such as the Vlasov-Poisson, Schrödinger-Poisson or time-dependent Hartree-Fock equations - are rigorously derived from first principles, i.e. from the fundamental microscopic models that govern the evolution of large, interacting particle systems.
\end{abstract}

Mon cher Athos, je veux bien, puisque votre santé l'exige absolument, que vous vous reposiez quinze jours. Allez donc prendre les eaux de Forges ou telles autres qui vous conviendront, et rétablissez-vous promptement. Votre affectionné

TRÉVILLE

Alexandre Dumas Les Trois Mousquetaires

\section{A review of physical models}

The subject matter of these lectures is the relation between "exact" microscopic models that govern the evolution of large particle systems and a certain type of approximate models known in Statistical Mechanics as "mean-field equations". This notion of mean-field equation is best understood by getting acquainted with the most famous examples of such equations, described below.

MSC 2000 : 82C05, 82C10, 82C22, 35A10, 35Q40, 35Q55.

Keywords : mean-field limit, Vlasov equation, Hartree approximation, Hartree-Fock equations, BBGKY hierarchy, propagation of chaos, Slater determinant. 


\subsection{Examples of mean-field equations}

Roughly speaking, a mean-field equation is a model that describes the evolution of a typical particle subject to the collective interaction created by a large number $N$ of other, like particles. The state of the typical particle is given by its phase space density; the force field exerted by the $N$ other particles on this typical particle is approximated by the average with respect to the phase space density of the force field exerted on that particle from each point in the phase space.

Here are the most famous examples of mean-field equations.

\subsubsection{The Vlasov equation}

The Vlasov equation is the kinetic model for collisionless gases or plasmas. Let $f \equiv f(t, x, \xi)$ be the phase space density of point particles that occupy position $x \in \mathbf{R}^{D}$ and have momentum $\xi \in \mathbf{R}^{D}$ at time $t \geq 0$. Let $V \equiv V(x, y)$ be the potential describing the pairwise interaction exerted by a particle located at position $y$ on a particle located at position $x$. Vlasov's equation reads

$$
\begin{array}{r}
\partial_{t} f+\frac{1}{m} \xi \cdot \nabla_{x} f+F(t, x) \cdot \nabla_{\xi} f=0, \quad x, \xi \in \mathbf{R}^{D} \\
F(t, x)=-\nabla_{x} \iint_{\mathbf{R}^{D} \times \mathbf{R}^{D}} V(x, y) f(t, y, \xi) d \xi d y .
\end{array}
$$

The parameter $m$ in the PDE above is the particle mass; below, we shall set it to be 1 without further apology, along with other physical constants.

In other words, Vlasov's equation can be put in the form of a Liouville equation

$$
\partial_{t} f+\left\{H_{f(t, \cdot, \cdot)}, f\right\}=0
$$

with a time-dependent Hamiltonian given by the formula

$$
H_{f(t, \cdot, \cdot)}(x, \xi)=\frac{1}{2 m}|\xi|^{2}+\iint_{\mathbf{R}^{D} \times \mathbf{R}^{D}} V(x, y) f(t, y, \xi) d \xi d y
$$

This formulation may be the best justification for the term "mean-field": the field exerted on the typical particle at time $t$ and position $x$ is created by the potential

$$
\int_{\mathbf{R}^{D}} V(x, y)\left(\int_{\mathbf{R}^{D}} f(t, x, \xi) d \xi\right) d y
$$

which is the sum of the elementary potentials created at position $x$ by one particle located at position $y$ distributed according to the macroscopic density

$$
\rho_{f}(t, x)=\int_{\mathbf{R}^{D}} f(t, x, \xi) d \xi
$$

The best known physical example of (1.1) (in space dimension $D=3$ ) is the case where

$$
V(x, y)=\frac{q}{4 \pi} \frac{1}{|x-y|} \quad \text { electrostatic potential created by a charge } q
$$


that is used to model collisionless plasmas with negligible magnetic effects. In truth, plasmas are made of several species of charged particles (electrons and ions); thus in reality the description of a plasma would involve as many transport equations in (1.1) as there are particle species to be considered, while the total electric force would be computed in terms of the sum of macroscopic densities of all particles weighted by the charge number of each particle species (e.g., if $q$ is minus the charge of one electron, $Z=-1$ for electrons, $Z=1,2, \ldots$ for positively charged ions...)

Another physical example of (1.1) is the case where

$$
V(x, y)=-\frac{G m}{|x-y|} \text { gravitational potential created by a mass } m
$$

where $G$ is Newton's constant. This model is used in Astronomy, in very specific contexts. Its mathematical theory is harder than that of the electrostatic case because the interaction between like particles in the present case is attractive (unlike in the previous case, where particle of like charges are repelled by the electrostatic force), which may lead to buildup of concentrations in the density.

Both models go by the name of "Vlasov-Poisson" equation, since $V$ is, up to a sign, the fundamental solution of Poisson's equation

$$
-\Delta_{x} U=\rho
$$

in $\mathbf{R}^{3}$.

There are many more physical examples of mean field equations of the Vlasov type than these two. For instance, one can replace the electrostatic interaction with the full electromagnetic interaction between charged, relativistic particles. The analogue of the Vlasov-Poisson equation in this case (in the somewhat unphysical situation where only one species of charged particles is involved) is

$$
\partial_{t} f+\frac{1}{m} \xi \cdot \nabla_{x} f+\frac{q}{m}\left(E+\frac{1}{c} v(\xi) \times B(t, x)\right) \cdot \nabla_{\xi} f=0
$$

coupled to the system of Maxwell's equations

$$
\begin{array}{ll}
\partial_{t} E-c \operatorname{curl}_{x} B=-j_{f}, & \operatorname{div}_{x} E=\rho_{f}, \\
\partial_{t} B+c \operatorname{curl}_{x} E=0, & \operatorname{div}_{x} B=0,
\end{array}
$$

where

$$
v(\xi)=\frac{\xi}{\sqrt{m^{2}+\frac{|\xi|^{2}}{c^{2}}}}, \quad j_{f}(t, x)=\int_{\mathbf{R}^{3}} v(\xi) f(t, x, \xi) d \xi .
$$

The system (1.4)-(1.5) is called "the relativistic Vlasov-Maxwell system". It is a good exercise to find its Hamiltonian formulation.

There are many more examples of Vlasov type equations, such as the VlasovEinstein equation which is the relativistic analogue of the gravitational VlasovPoisson equation above. There also exists a Vlasov-Yang-Mills equation for the quark-gluon plasma. 


\subsubsection{The mean-field Schrödinger equation}

Vlasov-type equations are classical, instead of quantum, models. There exist nonrelativistic, quantum analogues of Vlasov's equation (1.1). We shall be mainly concerned with two such models, the mean-field Schrödinger equation that is presented in this section, and the time-dependent Hartree-Fock equation, to be described in the next section.

The recipe for building the mean-field Schrödinger equation is to start from the time-dependent, classical mean-field Hamiltonian (1.2) in the form

$$
H_{\rho(t, \cdot)}(x, \xi)=\frac{1}{2 m}|\xi|^{2}+\int_{\mathbf{R}^{D}} V(x, y) \rho(t, y) d y
$$

and to quantize it by the usual rule $\xi \mapsto \frac{\hbar}{i} \nabla_{x}$, leading to the operator

$$
\mathcal{H}_{\rho(t, \cdot)}=-\frac{\hbar^{2}}{2 m} \Delta_{x}+\int_{\mathbf{R}^{D}} V(x, y) \rho(t, y) d y .
$$

If one considers a large number of undistinguishable quantum particles in the same "pure" state, the whole system of such particles is described by one wave function $\psi \equiv \psi(t, x) \in \mathbf{C}$, and the density $\rho$ is given by

$$
\rho(t, x)=|\psi(t, x)|^{2} .
$$

The evolution of the wave function $\psi$ is governed by the mean-field Schrödinger equation

$$
\begin{aligned}
i \hbar \partial_{t} \psi & =\mathcal{H}_{|\psi(t, \cdot)|^{2}} \psi \\
& =-\frac{\hbar^{2}}{2 m} \Delta_{x} \psi+\left(\int_{\mathbf{R}^{D}} V(x, y)|\psi(t, y)|^{2} d y\right) \psi
\end{aligned}
$$

It may also happen that the $N$ particles are in distinct quantum states, defined by a family of $N$ wave functions, $\psi_{k} \equiv \psi_{k}(t, x), k=1, \ldots, N$, that are orthonormal in $L^{2}$, i.e. $\left\langle\psi_{k} \mid \psi_{l}\right\rangle=\delta_{k l}$. In this case, the density $\rho$ is

$$
\rho(t, x)=\frac{1}{N} \sum_{k=1}^{N}\left|\psi_{k}(t, x)\right|^{2},
$$

and each wave function is governed by the Schrödinger equation defined by the Hamiltonian $\mathcal{H}_{\rho(t, \cdot)}$, i.e. the system

$$
\begin{array}{r}
i \hbar \partial_{t} \psi_{k}=-\frac{\hbar^{2}}{2 m} \Delta_{x} \psi_{k}+\left(\frac{1}{N} \sum_{k=1}^{N} \int_{\mathbf{R}^{D}} V(x, y)\left|\psi_{k}(t, y)\right|^{2} d y\right) \psi_{k}, \\
k=1, \ldots, N .
\end{array}
$$

One might think that the mean-field Schrödinger equation is a less universal object than its classical counterpart (the Vlasov equation) since there are so many variants of it - (1.6) and (1.7) for instance.

In fact, (1.6) and (1.7) (and all the other variants of the mean-field Schrödinger equation) are unified by considering instead the formulation of Quantum Mechanics 
in terms of operators. In this formulation, the state of this system is described by a time-dependent, integral operator $D(t) \in \mathcal{L}\left(L^{2}\left(\mathbf{R}^{D}\right)\right)$ - with integral kernel denoted by $D(t, x, y)$ — that satisfies the von Neumann equation

$$
i \hbar \partial_{t} D=\left[\mathcal{H}_{\rho(t, \cdot)}, D\right], \quad \text { where } \rho(t, x)=D(t, x, x) .
$$

The operator $D(t)$ is usually referred to as the density operator, while its integral kernel $D(t, x, y)$ is called the density matrix. A more explicit form of (1.8) is

$$
\begin{aligned}
i \hbar \partial_{t} D(t, x, y)= & -\frac{\hbar^{2}}{2 m}\left(\Delta_{x}-\Delta_{y}\right) D(t, x, y) \\
& +D(t, x, y) \int(V(x-z)-V(y-z)) D(t, z, z) d z
\end{aligned}
$$

In the case corresponding to the single mean-field Schrödinger equation (1.6), the density matrix is given by

$$
D(t, x, y)=\psi(t, x) \overline{\psi(t, y)}
$$

and the density operator it defines is usually denoted

$$
D(t)=|\psi(t, \cdot)\rangle\langle\psi(t, \cdot)|
$$

In the case corresponding to the system of $N$ coupled mean-field Schrö- dinger equations (1.7), the density matrix is given by

$$
D(t, x, y)=\frac{1}{N} \sum_{k=1}^{N} \psi_{k}(t, x) \overline{\psi_{k}(t, y)},
$$

while the density operator it defines is

$$
D(t)=\frac{1}{N} \sum_{k=1}^{N}\left|\psi_{k}(t, \cdot)\right\rangle\left\langle\psi_{k}(t, \cdot)\right| .
$$

Of course, in either case, the mean-field von Neumann equation (1.8) is equivalent to (1.6) or (1.7) - up to some unessential phase factor that may depend on $t$, the wave functions are recovered as the eigenfunctions of the density operator.

However, the mean-field von Neumann equation (1.8) does not take into account a purely quantum effet, the "exchange interaction", that is described in the next section.

\subsubsection{The (time-dependent) Hartree-Fock equation}

It is a well-known fact that the electrical interaction of (non-relativistic) charged particles is independent of their spins. However, the effect of the spin of non-relativistic particles is seen on their statistics. Indeed, one deduces from Relativistic Quantum Mechanics that particles with integer spin are bosons, meaning that the wave function of a system consisting of $N$ such particles is a symmetric function of their positions (see [20], §11). A similar argument shows that the wave function of a 
system of $N$ particles with half-integer spin is a skew-symmetric function of their positions (see [20], §25); in other words, such particles are fermions. For instance photons, $\alpha$ particles, hydrogen atoms, or $\pi$ mesons are bosons, while electrons, positrons, protons, neutrons etc... are fermions. That the wave function of a system of $N$ fermions is skew-symmetric implies Pauli's exclusion principle: two (or more) fermions cannot find themselves in the same quantum state. This apparently innocuous statement has far-reaching physical consequences - for instance on the structure of atoms, where successive electronic layers are filled with electrons of opposite spins.

Returning to the discussion in the previous section, one immediately sees that the mean-field Schrödinger equation (1.6), applies to bosons only, since it is a model based on the assumption that the $N$ particles are in the same quantum state characterized by the wave function $\phi$. This particular situation is referred to as "Bose condensation", and is typical for bosons at zero temperature.

Consider next a system consisting of 2 fermions: its wave function takes the form

$$
\frac{1}{\sqrt{2}}\left(\psi_{1}\left(x_{1}\right) \psi_{2}\left(x_{2}\right)-\psi_{1}\left(x_{2}\right) \psi_{2}\left(x_{1}\right)\right),
$$

where $\left(\psi_{1}, \psi_{2}\right)$ is an orthonormal system of $L^{2}\left(\mathbf{R}^{D}\right)$. If $V \equiv V\left(\left|x_{1}-x_{2}\right|\right)$ is a pairwise interaction potential, the interaction energy of this system is

$$
\begin{aligned}
& \iint V\left(x_{1}-x_{2}\right)\left|\psi_{1}\left(x_{1}\right)\right|^{2}\left|\psi_{2}\left(x_{2}\right)\right|^{2} d x_{1} d x_{2} \\
& \quad-\iint V\left(x_{1}-x_{2}\right) \psi_{1}\left(x_{1}\right) \overline{\psi_{1}\left(x_{2}\right)} \psi_{2}\left(x_{2}\right) \overline{\psi_{2}\left(x_{1}\right)} d x_{1} d x_{2} .
\end{aligned}
$$

In the expression above, the second integral is the "exchange interaction" (see [19], $\S 62$, problem 1).

From this elementary computation, one easily arrives at the mean-field equation for $N$ fermions: the $k$-th particle is subject to the sum of all interactions of the form (1.12) exerted by the $N-1$ other particles:

$$
\begin{aligned}
& i \hbar \partial_{t} \psi_{k}(t, x)=-\frac{\hbar^{2}}{2 m} \Delta \psi_{k}(t, x) \\
& +\frac{1}{N} \sum_{l=1}^{N} \psi_{k}(t, x) \int V(|x-y|)\left|\psi_{l}(t, y)\right|^{2} d y \\
& -\frac{1}{N} \sum_{l=1}^{N} \psi_{l}(t, x) \int V(|x-y|) \psi_{k}(t, y) \overline{\psi_{l}(t, y)} d y, \quad 1 \leq k \leq N .
\end{aligned}
$$

This system of equations is known as the "time dependent Hartree-Fock equations" (abbreviated as TDHF); the wave functions $\psi_{k}$ go by the name of "orbitals" of the system - we recall that the term "orbital" applies to that part of the wave function that does not depend on the spin variables.

Perhaps the simplest method for obtaining this system consists in writing the Hamiltonian as the sum of the kinetic energies of each particle, i.e.

$$
\sum_{k=1}^{N} \frac{\hbar^{2}}{2 m} \int\left|\nabla_{x} \psi_{k}(t, x)\right|^{2} d x,
$$


and of all the terms of the form (1.12) corresponding to each pair of particles. Then the evolution equation associated to the resulting Hamiltonian, say $\mathcal{H}$, is

$$
i \hbar \partial_{t} \psi_{k}=\frac{\delta \mathcal{H}}{\delta \overline{\psi_{k}(t, x)}} .
$$

The constant $1 / N$ on the right-hand side of (1.13) scales the interaction so that in the total energy (Hamiltonian $\mathcal{H}$ ), both the kinetic and interaction energies are of the same order of magnitude.

To emphasize the similarity between the TDHF equations and (1.9) — which may not seem that obvious on the formulation involving orbitals - let us write the operator form of (1.13). Let $D \equiv D(t, x, y)$ be the density matrix defined as in (1.11); assuming that the orbitals $\psi_{k}$ satisfy $(1.13), D(t, x, y)$ must satisfy

$$
\begin{aligned}
i \hbar \partial_{t} D(t, x, y)= & -\frac{\hbar^{2}}{2 m}\left(\Delta_{x}-\Delta_{y}\right) D(t, x, y) \\
& +\int(V(x-z)-V(y-z)) D(t, x, y) D(t, z, z) d z \\
& \left.-\int(V(x-z)-V(y-z)) D(t, x, z) D(t, z, y)\right) d z .
\end{aligned}
$$

In fact there are several variants of the TDHF equations presented here. In many applications (to Chemistry, for instance), they govern the evolution of electrons. In this case, the interaction potential $V$ involved in equations (1.13) is the Coulomb repulsive potential between negatively charged particles; it is also necessary to include other terms modelling the effect of the positively charged particles (nuclei, ions...). In most cases, including these terms does not result in additional mathematical difficulties, since they lead to linear perturbations of (1.13) in the electronic orbitals. For this reason, we shall restrict our attention to the simplified model (1.13) or (1.14) in this course.

\subsection{First principle equations}

Next we turn our attention to the fundamental equations describing the dynamics of a system of $N$ particles. In this course, we only consider non-relativistic particles. We are mainly concerned with quantum models; however, we also discuss the derivation of Vlasov's equation from the classical $N$-body problem, in the limit as $N \rightarrow+\infty$.

\subsubsection{The classical $N$-body problem}

Consider a system of $N$ point particles of mass $m$. In the absence of external forces (such as gravity), these particles are subject only to binary interactions corresponding to a potential $V \equiv V\left(x_{1}, x_{2}\right)$. (Obviously, $V$ is symmetric: $V\left(x_{1}, x_{2}\right)=$ $\left.V\left(x_{2}, x_{1}\right)\right)$. If $x_{k} \equiv x_{k}(t)$ denotes the position of the $k$-th particle, Newton's second law of motion for each particle is expressed in the form of the system of $N$ second-order differential equations:

$$
m \ddot{x}_{k}=-\sum_{1 \leq l \neq k \leq N} \nabla_{x_{k}} V\left(x_{k}, x_{l}\right), \quad 1 \leq k \leq N .
$$


Equivalently, one can write the Hamiltonian of this system of $N$ particles as the total energy $=$ kinetic energy + potential energy, i.e.

$$
H_{N}\left(x_{1}, \ldots, x_{N}, \xi_{1}, \ldots, \xi_{N}\right)=\sum_{k=1}^{N} \frac{\left|\xi_{k}\right|^{2}}{2 m}+\sum_{1 \leq l<k \leq N} V\left(x_{k}, x_{l}\right) .
$$

The system of second order differential equations (1.15) is equivalent to the system of Hamilton's equations

$$
\dot{x}_{k}=\nabla_{\xi_{k}} H_{N}, \quad \dot{\xi}_{k}=-\nabla_{x_{k}} H_{N} .
$$

The integral curves of this system of first-order differential equations are the characteristic curves of the Liouville equation

$$
\begin{aligned}
& \partial_{t} F_{N}+\left\{H_{N}, F_{N}\right\}:= \\
& \partial_{t} F_{N}+\sum_{k=1}^{N} \nabla_{\xi_{k}} H_{N} \cdot \nabla_{x_{k}} F_{N}-\sum_{k=1}^{N} \nabla_{x_{k}} H_{N} \cdot \nabla_{\xi_{k}} F_{N}=0
\end{aligned}
$$

In (1.18), $F_{N} \equiv F_{N}\left(t, x_{1}, \ldots, x_{N}, \xi_{1}, \ldots, \xi_{N}\right)$ can be seen as the joint probability density of the system of $N$ particles in the $N$-body phase space, i.e.

$$
F_{N}\left(t, x_{1}, \ldots, x_{N}, \xi_{1}, \ldots, \xi_{N}\right) d x_{1} \ldots d x_{N} d \xi_{1} \ldots d \xi_{N}
$$

is the probability that the particle 1 is to be found in a volume $d x_{1} d \xi_{1}$ of the 1particle phase space $\mathbf{R}^{D} \times \mathbf{R}^{D}$ around $\left(x_{1}, \xi_{1}\right)$, that the particle 2 is to be found in a volume $d x_{2} d \xi_{2}$ of the 1-particle phase space $\mathbf{R}^{D} \times \mathbf{R}^{D}$ around $\left(x_{2}, \xi_{2}\right)$, and so on. (In truth, the system of characteristics (1.17) can be used to propagate just any function $F_{N}$ on the $N$-body phase space, but the only such function that is physically meaningful is the joint probability density described above).

\subsubsection{The quantum $N$-body problem}

Next we describe the quantum analogue of (1.15). The quickest route to this model is to quantize the Hamiltonian $H_{N}$ in (1.16), as we already did for the mean-field Hamiltonian. Here, quantization means associating to a function defined on the $N$ body phase space $\left(\mathbf{R}_{x}^{D} \times \mathbf{R}_{\xi}^{D}\right)^{N}$ an operator acting on the Hilbert space $L^{2}\left(\left(\mathbf{R}_{x}^{D}\right)^{N}\right)$ - here, $\left(\mathbf{R}_{x}^{D}\right)^{N}$ is usually called the configuration space. For an arbitrary function, there is more than one way of doing this; but for a function such as $H_{N}$, quantization means replacing the momentum variable $\xi_{k}$ with the momentum operator $\frac{\hbar}{i} \nabla_{x_{k}}$. Therefore, the Hamiltonian function $H_{N}$ is transformed into the Hamiltonian operator

$$
\mathcal{H}_{N}=\sum_{k=1}^{N}-\frac{\hbar^{2}}{2 m} \Delta_{x_{k}}+\sum_{1 \leq k<l \leq N} V\left(x_{k}, x_{l}\right) .
$$

With the Hamiltonian (1.19), one writes the $N$-body Schrödinger equation

$$
\begin{aligned}
i \hbar \partial_{t} \Psi_{N} & =\mathcal{H}_{N} \Psi_{N} \\
& =\sum_{k=1}^{N}-\frac{\hbar^{2}}{2 m} \Delta_{x_{k}} \Psi_{N}+\sum_{1 \leq k<l \leq N} V\left(x_{k}, x_{l}\right) \Psi_{N} .
\end{aligned}
$$


Here $\Psi_{N} \equiv \Psi_{N}\left(t, x_{1}, \ldots, x_{N}\right)$ is the wave-function of the system of $N$ particles.

The analogue of the classical, $N$-body Liouville equation (1.18) is the $N$-body von Neumann equation

$$
\begin{aligned}
i \hbar \partial_{t} D_{N}\left(t, X_{N}, Y_{N}\right) & =\left[\mathcal{H}_{N}, D_{N}\right]\left(t, X_{N}, Y_{N}\right) \\
& =\sum_{k=1}^{N}-\frac{\hbar^{2}}{2 m}\left(\Delta_{x_{k}}-\Delta_{y_{k}}\right) D_{N}\left(t, X_{N}, Y_{N}\right) \\
& +\sum_{1 \leq k<l \leq N}\left(V\left(x_{k}, x_{l}\right)-V\left(y_{k}, y_{l}\right)\right) D_{N}\left(t, X_{N}, Y_{N}\right),
\end{aligned}
$$

with the notation $X_{N}=\left(x_{1}, \ldots, x_{N}\right), Y_{N}=\left(y_{1}, \ldots, y_{N}\right)$, where the density matrix is defined in terms of the wave-function by the formula

$$
D_{N}\left(t, X_{N}, Y_{N}\right)=\Psi_{N}\left(t, X_{N}\right) \overline{\Psi_{N}\left(t, Y_{N}\right)} .
$$

It is left as an easy exercise to check that, if $\Psi_{N}$ is a solution of the Schrödinger equation (1.20), then the density matrix defined by the formula above is a solution of the von Neumann equation (1.21).

\subsection{Another example of mean-field limit}

There is another classical example of mean-field limit, namely the vorticity formulation of the two-dimensional Euler equation of perfect, incompressible fluids. Let $u(t, x)=\left(u_{1}\left(t, x_{1}, x_{2}\right), u_{2}\left(t, x_{1}, x_{2}\right)\right)$ be the velocity field and $\omega=\left(\partial_{x_{1}} u_{2}-\partial_{x_{2}} u_{1}\right)$. The Euler equation is

$$
\partial_{t} u+\left(u \cdot \nabla_{x}\right) u+\nabla_{x} p=0, \quad \operatorname{div} u=0 .
$$

Taking the curl of the equation above leads to

$$
\partial_{t} \omega+\operatorname{div}_{x}(u \omega)=0, \quad u=\nabla_{x}^{\perp} \Delta^{-1} \omega
$$

where the orthogonal gradient is defined as $\nabla^{\perp}=\left(-\partial_{x_{2}}, \partial_{x_{1}}\right)$. The operator $\nabla_{x}^{\perp} \Delta^{-1}$ - say, on $\mathbf{T}^{2}$ - is an integral operator of the form

$$
u(t, x)=(K \star \omega(t, \cdot))(x),
$$

where $K$ is the orthogonal gradient of the fundamental solution of $\Delta$, so that the vorticity formulation above of the Euler equation is recast as

$$
\partial_{t} \omega+\operatorname{div}_{x}((K \star \omega(t, \cdot)) \omega)=0 .
$$

This is another example of mean-field PDE, very similar to the Vlasov-Poisson equation. In his famous article [28], Onsager got the idea of explaining two-dimensional turbulence by the methods of Statistical Mechanics applied to (1.22), viewed as the mean-field limit of a system of point vortices of equal strengths located at the positions $z_{1}(t), \ldots, z_{N}(t)$ satisfying the system of ODEs

$$
\dot{z_{k}}=\frac{1}{N} \sum_{1 \leq l \neq k \leq N} K\left(Z_{k}(t)-z_{l}(t)\right), \quad 1 \leq k \leq N .
$$


We refer to [9] and to [17] for a mathematical treatment of some of the main ideas in Onsager's paper.

This description is also used to design numerical methods for the two-dimensional Euler equations (the so-called vortex method): we refer the interested reader to the book by Marchioro-Pulvirenti [23] and to the references therein.

However, we shall not insist too much on this theme in these lectures, since the $N$-vortex system (1.23) is not a fundamental principle of physics, but rather an approximation of the physical model (1.22). Yet, in view of the simplicity of this model, we shall use it instead of Vlasov's equation in Appendices A-B to part I of these lectures, to show Dobrushin's stability estimate in terms of the Wasserstein distance, with its application to the propagation of chaos.

\subsection{The Boltzmann equation is not a mean-field equation}

Vlasov's equation belongs to the kinetic theory of plasmas. Indeed, it governs the 1-particle phase space density of a system consisting of a large number of like point particles, which is precisely the fundamental notion that appeared in the founding papers by Maxwell and Boltzmann.

However, not every kinetic model is a mean-field equation. The most famous of such models, the Boltzmann equation itself, is not. It reads

$$
\left(\partial_{t}+v \cdot \nabla_{x}\right) f=\iint_{\mathbf{R}^{3} \times \mathbf{S}^{2}}\left(f^{\prime} f_{1}^{\prime}-f f_{1}\right)|v \cdot \omega| d v_{1} d \omega
$$

where $f \equiv f(t, x, v)$ is the probability density of particles which, at time $t$, are at position $x$ with velocity $v$. The notations $f_{1}, f^{\prime}$ and $f_{1}^{\prime}$ designate respectively $f\left(t, x, v_{1}\right), f\left(t, x, v^{\prime}\right)$, and $f\left(t, x, v_{1}^{\prime}\right)$, where

$$
v^{\prime}=v-\left(v-v_{1}\right) \cdot \omega \omega, \quad v_{1}^{\prime}=v_{1}+\left(v-v_{1}\right) \cdot \omega \omega .
$$

It is interesting to compare the Vlasov equation (1.1) with (1.24). Since the momentum $\xi=m v$ where $m$ is the particle mass, this amounts to comparing

$$
-F \cdot \nabla_{\xi} f \quad \text { with } \quad \iint_{\mathbf{R}^{3} \times \mathbf{S}^{2}}\left(f^{\prime} f_{1}^{\prime}-f f_{1}\right)|v \cdot \omega| d v_{1} d \omega .
$$

The latter term, which is Boltzmann's collision integral, describes the loss of particles with velocity $v$ due to binary collisions with particles located at the same position $x$ with an arbitrary velocity $v_{1}$, and the creation of particles with that velocity $v$ due to binary collisions involving one particle with velocity $v^{\prime}$ and another particle with velocity $v_{1}^{\prime}$, both located at that same position $x$. Here, particles are viewed as hard spheres with radius 0 and the collisions are assumed to be perfectly elastic. Hence, each particle is unaffected by all the other particles, unless it touches another particle, in which case a collision of the type described above occurs. Because the radius of these particles is negligible - or 0 - collisions involving more than two particles are infinitely rare events and are discarded in Boltzmann's equation.

By constrast, the former term, i.e. the mean-field term in Vlasov's equation, takes into account the effect on any given particle of all the other particles, and not 
only the effect of its closest neighbor. In this sense, one could think that Vlasov's equation is better adapted at describing long-range interactions, while Boltzmann's equation is better suited for short-range interactions. There is some truth in such a statement, which however fails to capture the essential differences between both models.

In fact, Boltzmann's equation for hard spheres was rigorously derived by Lanford [22] from the model analogous to (1.15) where the potential $V$ is replaced with hard sphere collisions, under some scaling assumption known as the Boltzmann-Grad scaling. There is an unpublished proof by King [18] that does the same for some class of short-range potentials. Vlasov's equation is also derived from (1.15), but under a very different scaling assumption.

Rather strikingly, both limits lead to very different qualitative behaviors. For instance, it is believed (and indeed proved in a variety of cases by Desvillettes and Villani [13]) that the long time limit of the Boltzmann equation in a compact domain (e.g. on a flat 3-torus) leads to uniform Maxwellian distributions (i.e. Gaussian distributions in the $v$ variable with moments that are constant in the variable $x$ ). Boltzmann's H-Theorem states that, if $f$ is a solution to the Boltzmann equation, the quantity

$$
-\iint f(t, x, v) \ln f(t, x, v) d x d v
$$

(which represents some form of entropy) increases until $f$ reaches a Maxwellian equilibrium. This is related to the notion of irreversibility in Statistical Mechanics, however in a way that was very misleading in Boltzmann's time, thereby leading to bitter controversy.

On the contrary, this quantity is a constant if $f$ is a solution to the Vlasov equation - this is left as an easy exercise, whose solution is reminiscent of the proof of Liouville's theorem on Hamilton's equation (i.e. the invariance of the measure $d x d \xi$ under the Hamiltonian flow). Moreover, the long time limit of the solution of Vlasov's equation on a compact domain, say on a flat torus, is not yet understood, and seems a rather formidable open problem. By the way, it is interesting to note that the quantum analogue of (1.25) is

$$
\operatorname{trace}(D(t) \ln D(t))
$$

where $D \equiv D(t)$ is the density operator - notice that $D(t) \ln D(t)$ is well defined by the continuous functional calculus, since $D(t)$ is a positive self-adjoint operator and $z \mapsto z \ln z$ a continuous function on $\mathbf{R}_{+}$.

\section{OUtLine}

Part I: From the Classical $N$-body problem to Vlasov's equation

Part II: From the quAntum $N$-Body Problem to Hartree's Equation

PART III: From the QUANTUM $N$-Body Problem to The TDHF EQUATIONS 


\section{Part I. From the classical $N$-body problem to Vlasov's equa- tion}

In this section, we start from the classical $N$-body problem in Hamiltonian form:

$$
\begin{aligned}
& \dot{x}_{k}=\xi_{k}, \\
& \dot{\xi}_{k}=-\frac{1}{N} \sum_{1 \leq k \neq l \leq N} \nabla_{x} V\left(x_{k}, x_{l}\right)
\end{aligned}
$$

which is equivalent to (1.17) with a Hamiltonian

$$
H_{N}\left(x_{1}, \ldots, x_{N}, \xi_{1}, \ldots, \xi_{N}\right)=\sum_{k=1}^{N} \frac{1}{2}\left|\xi_{k}\right|^{2}+\frac{1}{N} \sum_{1 \leq l<k \leq N} V\left(x_{k}, x_{l}\right)
$$

where the interaction potential $V \equiv V(x, y)$ is scaled by the $1 / N$ factor.

This situation is known as "the weak coupling scaling"; it naturally leads to mean-field equations, since the force exerted on each particle is of order one as $N \rightarrow+\infty$.

Perhaps the most convincing argument in favor of this scaling is as follows: since the total kinetic energy is a sum of $N$ terms and the total potential energy is a sum of $\frac{1}{2} N(N-1)$ terms, the $1 / N$ factor in multiplying the potential scales the kinetic and potential energy in the Hamiltonian to be of the same order of magnitude as $N \rightarrow+\infty$.

For notational simplicity, we set $z_{k}=\left(x_{k}, \xi_{k}\right) \in \mathbf{R}^{D} \times \mathbf{R}^{D}$, with $k=1, \ldots, N$. The potential $V: \mathbf{R}^{D} \times \mathbf{R}^{D} \rightarrow \mathbf{R}$ is assumed to satisfy

$$
V \in C_{b}^{2}\left(\mathbf{R}^{D} \times \mathbf{R}^{D}\right), \quad V(x, y)=V(y, x) \text { for all } x, y \in \mathbf{R}^{D} .
$$

Under this assumption, the Hamiltonian vector field

$$
Z \mapsto\left(\nabla_{x_{k}} H_{N}(Z),-\nabla_{\xi_{k}} H_{N}(Z)\right)_{1 \leq k \leq N}^{T}
$$

belongs to $C_{b}^{1}\left(\left(\mathbf{R}^{D} \times \mathbf{R}^{D}\right)^{N},\left(\mathbf{R}^{D} \times \mathbf{R}^{D}\right)^{N}\right)$.

By the Cauchy-Lipschitz theorem, for each $Z_{N}^{0} \in\left(\mathbf{R}^{D} \times \mathbf{R}^{D}\right)^{N}$, there exists a unique solution $Z_{N}(t)=\left(x_{k}(t), \xi_{k}(t)\right)_{1 \leq k \leq N}$ to the system of ODEs (1.17) such that $Z_{N}(0)=Z_{N}^{0}$. This solution is denoted by $Z_{N} \equiv Z_{N}\left(t, Z_{N}^{0}\right)$ and is defined for all times. Finally, $Z_{N}$ belongs to $C^{1}\left(\mathbf{R} \times\left(\mathbf{R}^{D} \times \mathbf{R}^{D}\right)^{N} ;\left(\mathbf{R}^{D} \times \mathbf{R}^{D}\right)^{N}\right)$.

Definition I.1. The empirical distribution of the system of $N$ particles is the probability measure on $\mathbf{R}^{D} \times \mathbf{R}^{D}$ defined as

$$
f_{N, Z_{N}^{0}}(t, \cdot)=\frac{1}{N} \sum_{k=1}^{N} \delta_{z_{k}\left(t, Z_{N}^{0}\right)}
$$

This is a probability measure on the 1-particle phase space, and yet, it is equivalent to know $f_{N, Z_{N}^{0}}$ or to know the trajectory of each of the particles considered. This probability measure is not to be confused with the unknown in the Liouville equation (1.18), the latter being a probability measure on the $N$-particle phase space. 
We shall discuss the relation between these two probability measures in Appendix B below.

For the time being, let us point at a nice feature of the empirical distribution: like the solution $f$ of the Vlasov's equation - the target equation to be derived from (1.17) — it is already defined on the 1-particle phase space, unlike the solution of the Liouville equation (1.18), that lives in a different, bigger space.

In fact, $f_{N, Z_{N}^{0}}$ is a solution - in fact an approximate solution - to (1.1) in the sense of distributions, and this statement is equivalent to the fact that $\left(x_{k}, \xi_{k}\right)$ is a solution to (1.17).

Lemma I.2. Let $Z_{N} \equiv Z_{N}\left(t, Z_{N}^{0}\right)$ be the solution of (I.1) with initial data $Z_{N}\left(0, Z_{N}^{0}\right)=$ $Z_{N}^{0}$. Then the empirical distribution $f_{N, Z_{N}^{0}}$ satisfies

$$
\begin{aligned}
\partial_{t} f_{N, Z_{N}^{0}}+\xi \cdot \nabla_{x} f_{N, Z_{N}^{0}} & +\operatorname{div}_{\xi}\left(F_{N, Z_{N}^{0}}(t, x) f_{N, Z_{N}^{0}}\right) \\
& =\frac{1}{N^{2}} \sum_{k=1}^{N} \operatorname{div}_{\xi}\left(\nabla_{x} V\left(x_{k}, x_{k}\right) \delta_{z_{k}\left(t, Z_{N}^{0}\right)}\right)
\end{aligned}
$$

in the sense of distributions, where

$$
F_{N, Z_{N}^{0}}(t, x)=-\nabla_{x} \iint V(x, y) f_{N, Z_{N}^{0}}(t, d y, d \xi)
$$

(In (I.4), $\nabla_{x} V$ designates the derivative with respect to the first group of variables in the product space $\left.\mathbf{R}^{D} \times \mathbf{R}^{D}\right)$.

Proof. Let $\phi \equiv \phi(x, \xi) \in C_{c}^{\infty}\left(\mathbf{R}^{D} \times \mathbf{R}^{D}\right)$. Then

$$
\begin{aligned}
\partial_{t}\left\langle f_{N, Z_{N}^{0}}(t), \phi\right\rangle & =\frac{1}{N} \sum_{k=1}^{N} \dot{x}_{k}(t) \cdot \nabla_{x} \phi\left(x_{k}(t), \xi_{k}(t)\right) \\
& +\frac{1}{N} \sum_{k=1}^{N} \dot{\xi}_{k}(t) \cdot \nabla_{\xi} \phi\left(x_{k}(t), \xi_{k}(t)\right) \\
& =\frac{1}{N} \sum_{k=1}^{N} \xi_{k}(t) \cdot \nabla_{x} \phi\left(x_{k}(t), \xi_{k}(t)\right) \\
& +\frac{1}{N} \sum_{k=1}^{N} F_{N, Z_{N}^{0}}\left(t, x_{k}(t)\right) \cdot \nabla_{\xi} \phi\left(x_{k}(t), \xi_{k}(t)\right) \\
& -\frac{1}{N^{2}} \sum_{k=1}^{N} \nabla_{x} V\left(x_{k}(t), x_{k}(t)\right) \cdot \nabla_{\xi} \phi\left(x_{k}(t), \xi_{k}(t)\right)
\end{aligned}
$$

which gives the announced equality.

Next we study the limiting behavior of $f_{N, Z_{N}^{0}}$ as $N \rightarrow+\infty$; some uniform estimates are needed for this. In fact we need only the conservation of Hamilton's function under the Hamiltonian flow (1.17), expressed in the following manner: 
Lemma I.3. Let $Z_{N}^{0}=\left(x_{k}^{0}, \xi_{k}^{0}\right)_{1 \leq k \leq N}$. Assume that

$$
\sup _{\substack{1 \leq k \leq N \\ N \geq 1}}\left|\xi_{k}^{0}\right|<+\infty
$$

Then

$$
\sup _{\substack{N \geq 1 \\ t \in \mathbf{R}}} \iint|\xi|^{2} f_{N, Z_{N}^{0}}(t, x, \xi) d x d \xi<+\infty .
$$

Proof. The Hamiltonian $H_{N}$ is constant on the integral curves of the associated Hamiltonian vector field; hence

$$
\frac{1}{N} H_{N}\left(Z_{N}\left(t, Z_{N}^{0}\right)\right)=\frac{1}{N} H_{N}\left(Z_{N}^{0}\right) \leq \frac{1}{2} \sup _{1 \leq k \leq N, N \geq 1}\left|\xi_{k}^{0}\right|^{2}+\frac{1}{2}\|V\|_{L^{\infty}}
$$

On the other hand, one has

$$
\begin{aligned}
\iint|\xi|^{2} f_{N, Z_{N}^{0}}(t, x, \xi) d x d \xi & =\frac{1}{N} \sum_{k=1}^{N}\left|\xi_{k}(t)\right|^{2} \\
& \leq \frac{2}{N} H_{N}\left(Z_{N}\left(t, Z_{N}^{0}\right)\right)+\|V\|_{L^{\infty}}
\end{aligned}
$$

which implies the announced result.

Lemma I.4. Let $V \in C_{r}^{2}\left(\mathbf{R}^{D} \times \mathbf{R}^{D}\right)^{1}$. Let $Z_{N}^{0}$ be such that

$$
f_{N, Z_{N}^{0}}(0)=\frac{1}{N} \sum_{k=1}^{N} \delta_{\left(Z_{N}^{0}\right)_{k}} \rightarrow f^{0}
$$

weakly- ${ }^{*}$ in $\mathcal{M}\left(\mathbf{R}^{D} \times \mathbf{R}^{D}\right)^{2}$ and

$$
H_{N}\left(Z_{N}^{0}\right)=O(N)
$$

as $N \rightarrow+\infty$. Then, the sequence $f_{N, Z_{N}^{0}}$ is relatively compact in $C\left(\mathbf{R}_{+} ; w^{*}-\mathcal{M}\left(\mathbf{R}^{D} \times\right.\right.$ $\mathbf{R}^{D}$ )) (for the topology of uniform convergence on compact subsets of $\mathbf{R}_{+}$) and each of its limit points as $N \rightarrow+\infty$ is a solution $f$ to the Vlasov equation

$$
\begin{gathered}
\partial_{t} f+\xi \cdot \nabla_{x} f+F(t, x) \cdot \nabla_{\xi} f=0, \quad x, \xi \in \mathbf{R}^{D}, \\
F(t, x)=-\nabla_{x} \iint_{\mathbf{R}^{D} \times \mathbf{R}^{D}} V(x, y) f(t, y, \xi) d \xi d y .
\end{gathered}
$$

in the sense of distributions, with initial data

$$
\left.f\right|_{t=0}=f^{0} .
$$

\footnotetext{
${ }^{1}$ We denote by $C_{r}^{k}\left(\mathbf{R}^{N}\right)$ the space of $C^{k}$ functions $f$ on $\mathbf{R}^{D}$ such that, for all $n \geq 0$ and all $p=1, \ldots, k,\left|D^{p} f(x)\right|=O\left(|x|^{-n}\right)$ as $|x| \rightarrow+\infty$.

${ }^{2}$ We denote by $\mathcal{M}(X)$ the space of Radon measures on a locally compact space $X$; its weak-* topology is the one defined by duality with test functions in $C_{c}(X)$.
} 
Proof. For each $t \geq 0, f_{N, Z_{N}^{0}}(t)$ is a sequence of probability measures on $\mathbf{R}^{D} \times \mathbf{R}^{D}$. Hence $f_{N, Z_{N}^{0}}$ is weakly-* relatively compact in $\mathcal{M}\left(\mathbf{R}_{+} \times \mathbf{R}^{D} \times \mathbf{R}^{D}\right)$. Denote by $f_{N^{\prime}, Z_{N^{\prime}}^{0}}$ be a subsequence of $f_{N, Z_{N}^{0}}$ weakly-* converging to $f$. Because of Lemma I.3,

$$
\int f_{N^{\prime}, Z_{N^{\prime}}^{0}}(t, x, \xi) d \xi \rightarrow \int f(t, x, \xi) d \xi
$$

weakly- ${ }^{*}$ in $\mathcal{M}\left(\mathbf{R}_{+} \times \mathbf{R}^{D}\right)$ as $N^{\prime} \rightarrow+\infty$.

On the other hand, any solution to the Hamiltonian system (I.1) satisfies the bound

$$
\sup _{\substack{t \geq 0 \\ 1 \leq k \leq N}}\left|\ddot{x}_{k}(t)\right| \leq\left\|\nabla_{x} V\right\|_{L^{\infty}} .
$$

From this bound, an easy argument shows that, for each $\phi \in C_{c}\left(\mathbf{R}^{D} \times \mathbf{R}^{D}\right)$, the sequence of functions

$$
t \mapsto\left\langle f_{N^{\prime} Z_{N^{\prime}}^{0}}(t), \phi\right\rangle
$$

is equicontinuous on compact subsets of $\mathbf{R}_{+}$. By Ascoli's theorem

$$
F_{N^{\prime}, Z_{N^{\prime}}^{0}} \rightarrow \iint-\nabla_{x} V(x, y) f(t, y, \eta) d \eta d y
$$

uniformly on compact subsets of $\mathbf{R}_{+} \times \mathbf{R}^{D}$ as $N^{\prime} \rightarrow+\infty$. Thus

$$
F_{N^{\prime}, Z_{N^{\prime}}^{0}} f_{N^{\prime}, Z_{N^{\prime}}^{0}} \rightarrow f(t, x, \xi) \iint-\nabla_{x} V(x, y) f(t, y, \eta) d \eta d y
$$

in the sense of distributions on $\mathbf{R}_{+} \times \mathbf{R}^{D} \times \mathbf{R}^{D}$. Hence $f$ solves Vlasov's equation (1.1) in the sense of distributions. Also, $\left.f\right|_{t=0}=f^{0}$ because of the equicontinuity of (I.6).

Notice that classical solutions to the Vlasov equation (I.5) are unique- ly defined by their initial data, as shown by the following

Lemma I.5. Let $V \in C_{r}^{2}\left(\mathbf{R}^{D} \times \mathbf{R}^{D}\right)$, and let $f^{0} \in C^{1}\left(\mathbf{R}^{D} \times \mathbf{R}^{D}\right)$ be such that

$$
f^{0} \geq 0, \quad \iint(1+|\xi|)^{2} f^{0}(x, \xi) d x d \xi,+\infty .
$$

Then the Vlasov equation (I.5) has a unique classical solution $f \in C^{1}\left(\mathbf{R}_{+} \times \mathbf{R}^{D} \times\right.$ $\mathbf{R}^{D}$ ) with initial data

$$
\left.f\right|_{t=0}=f^{0} .
$$

Any $g \in C\left(\mathbf{R}_{+}, w^{*}-\mathcal{M}\left(\mathbf{R}^{D} \times \mathbf{R}^{D}\right)\right)$ that solves (1.1) in the sense of distributions on $\mathbf{R}_{+}^{*} \times \mathbf{R}^{D} \times \mathbf{R}^{D}$ and satisfies $\left.g\right|_{t=0}=f^{0}$ must coincide with $f$ on all of $\mathbf{R}_{+}^{*} \times \mathbf{R}^{D} \times \mathbf{R}^{D}$.

The (easy) proof of this Lemma is left to the reader (we shall prove a slightly more general result later).

Collecting the results in Lemmas I.4 and I.5, we arrive at the following Theorem, proved by several authors (Neunzert [26], Braun-Hepp [8], Dobrushin [14] and Maslov [24]). 
Theorem I.6. Let $V \in C_{r}^{2}\left(\mathbf{R}^{D} \times \mathbf{R}^{D}\right)$. Start from a sequence $Z_{N}^{0, N}$ of initial configurations of $N$ particles such that

$$
\frac{1}{N} \sum_{k=1}^{N} \delta_{\left(Z_{N}^{0, N}\right)_{k}} \rightarrow f^{0} \text { in } w^{*}-\mathcal{M}\left(\mathbf{R}^{D} \times \mathbf{R}^{D}\right)
$$

and

$$
H_{N}\left(Z_{N}^{0, N}\right)=O(N)
$$

Then

$$
\frac{1}{N} \sum_{k=1}^{N} \delta_{z_{k}\left(t, Z_{N}^{0, N}\right)} \rightarrow f(t, \cdot, \cdot) \text { in } w^{*}-\mathcal{M}\left(\mathbf{R}^{D} \times \mathbf{R}^{D}\right)
$$

uniformly on compacts subsets of $\mathbf{R}_{+}$, where $f$ is the solution to the Vlasov equation (1.1) with initial data $f^{0}$.

Hence, Vlasov's equation (1.1) is shown to be the mean-field limit of the $N$-body Hamiltonian system (1.17) as $N \rightarrow+\infty$.

In fact the proof given here reduces to the continuous dependence on initial data of the solution to Vlasov's equation.

\section{I.A. Mean-field PDEs and Wasserstein distance}

In this first appendix to Part I, we present Dobrushin's beautiful idea of proving stability of first order mean-field PDEs in the Wasserstein distance.

Definition I.7. Let $\mu$ and $\nu$ be two probability measures on $\mathbf{R}^{D}$. The Wasserstein distance between $\mu$ and $\nu$ is

$$
W(\mu, \nu)=\inf \{\mathbf{E}|X-Y| \mid X(\text { resp. } Y) \text { has distribution } \mu(\text { resp. } \nu)\}
$$

In other words, let $E(\mu, \nu)$ be the set of probability measures $P$ on $\mathbf{R}^{D} \times \mathbf{R}^{D}$ such that the image of $P$ under the projection on the first factor (resp. on the second factor) is $\mu$ (resp. $\nu$ ). Then

$$
W(\mu, \nu)=\inf _{P \in E(\mu, \nu)} \iint|x-y| P(d x d y) .
$$

Consider next the mean-field, 1st order PDE, for the unknown $\rho \equiv \rho(t, z)$,

$$
\partial_{t} \rho+\operatorname{div}_{z}\left(\left(K \star_{z} \rho\right) \rho\right)=0, \quad \rho_{\mid t=0}=\rho^{0} .
$$

where $K$ is a Lipschitz continuous vector-field on $\mathbf{R}^{D}$ and $\star_{z}$ denotes the convolution in $z$. This is model is analogous to the vorticity formulation of the two-dimensional Euler equation (see section 1 above), except that the function $K$ in that case is singular at the origin. In the context of vortex method for the Euler equation, it is customary to replace the original kernel $K$ by a truncation of it near the origin — this is called the "vortex blob" method, since it amounts to assign a positive thickness to each vortex center in the system considered: see [23]. 
The associated system of characteristics is

$$
\begin{aligned}
& \dot{z}\left(t, a, \rho^{0}\right)=\left(K \star_{z} \rho(t)\right)\left(z\left(t, a, \rho^{0}\right)\right), \\
& z\left(0, a, \rho^{0}\right)=a, \quad \rho(t)=z\left(t, \cdot, \rho^{0}\right)_{*} \rho^{0} .
\end{aligned}
$$

(We recall the following usual notation for transportation of measure: given two measurable spaces $(X, \mathcal{M})$ and $(Y, \mathcal{N})$, a measurable map $f: X \rightarrow Y$ and a measure $\mu$ on $(X, \mathcal{M})$, one defines a measure $\nu$ on $(Y, \mathcal{N})$ called the "image of $\mu$ by $f$ " and denoted by $\nu=f_{*} \mu$, by the formula $\nu(B)=\mu\left(f^{-1}(B)\right)$ for $\left.B \in \mathcal{N}\right)$. In other words

$$
\dot{z}\left(t, a, \rho^{0}\right)=\int K\left(z\left(t, a, \rho^{0}\right)-z\left(t, a^{\prime}, \rho^{0}\right)\right) \rho^{0}\left(d a^{\prime}\right) .
$$

Let $\mu \equiv \mu(t, d z)$ and $\nu \equiv \nu(t, d z)$ be two solutions to the mean-field PDE (I.7) in $C\left(\mathbf{R}_{+}, w^{*}-\mathcal{M}^{1}\left(\mathbf{R}^{D}\right)\right)^{3}$ Then

\section{DOBRUSHIN'S INEQUALITY}

$$
W(\mu(t), \nu(t)) \leq W\left(\mu^{0}, \nu^{0}\right) e^{2 t\|K\|_{L i p}}, \quad t \geq 0 .
$$

Proof. Let $P^{0} \in E\left(\mu^{0}, \nu^{0}\right)$; define $P(t)$ to be the image of $P^{0}$ under the map $(a, b) \mapsto$ $\left(z\left(t, a, \mu^{0}\right), z\left(t, b, \nu^{0}\right)\right)$. Then $P(t) \in E(\mu(t), \nu(t))$. Set

$$
\Phi(t)=\iint|a-b| P(t, d a d b)=\iint\left|z\left(t, a, \mu^{0}\right)-z\left(t, b, \nu^{0}\right)\right| P^{0}(d a d b) .
$$

Observe that

$$
\begin{aligned}
z\left(t, a, \mu^{0}\right) & =a+\int_{0}^{t} \int K\left(z\left(s, a, \mu^{0}\right)-z\left(s, a^{\prime}, \mu^{0}\right)\right) \mu^{0}\left(d a^{\prime}\right) d s \\
& =a+\int_{0}^{t} \iint K\left(z\left(s, a, \mu^{0}\right)-z\left(s, a^{\prime}, \mu^{0}\right)\right) P^{0}\left(d a^{\prime} d b^{\prime}\right) d s
\end{aligned}
$$

and similarly

$$
\begin{aligned}
z\left(t, b, \nu^{0}\right) & =b+\int_{0}^{t} \int K\left(z\left(s, b, \nu^{0}\right)-z\left(s, b^{\prime}, \nu^{0}\right)\right) \nu^{0}\left(d b^{\prime}\right) d s \\
& =b+\int_{0}^{t} \iint K\left(z\left(s, b, \nu^{0}\right)-z\left(s, b^{\prime}, \nu^{0}\right)\right) P^{0}\left(d a^{\prime} d b^{\prime}\right) d s,
\end{aligned}
$$

so that

$$
\begin{aligned}
& \left|z\left(t, a, \mu^{0}\right)-z\left(t, b, \nu^{0}\right)\right| \leq|a-b| \\
& +\int_{0}^{t} \iint\|K\|_{L i p} \mid\left(z\left(s, a, \mu^{0}\right)-z\left(s, a^{\prime}, \mu^{0}\right)\right) \\
& -\left(z\left(s, b, \nu^{0}\right)-z\left(s, b^{\prime}, \nu^{0}\right)\right) \mid P^{0}\left(d a^{\prime} d b^{\prime}\right) d s \\
& \left.\leq|a-b|+\|K\|_{\text {Lip }} \int_{0}^{t} \mid z\left(s, a, \mu^{0}\right)-z\left(s, b, \nu^{0}\right)\right) \mid d s \\
& \left.+\|K\|_{\text {Lip }} \int_{0}^{t} \iint \mid z\left(s, a^{\prime}, \mu^{0}\right)-z\left(s, a^{\prime}, \nu^{0}\right)\right) \mid P^{0}\left(d a^{\prime} d b^{\prime}\right) d s .
\end{aligned}
$$

\footnotetext{
${ }^{3} \mathcal{M}^{1}(X)$ denotes the set of probability measures on $X$.
} 
Integrating both sides of this inequality with respect to $P^{0}(d a d b)$ leads to

$$
\begin{aligned}
& \iint\left|z\left(t, a, \mu^{0}\right)-z\left(t, b, \nu^{0}\right)\right| P^{0}(d a d b) \leq \iint|a-b| P^{0}(d a d b) \\
& \left.+\|K\|_{L i p} \int_{0}^{t} \iint \mid z\left(s, a, \mu^{0}\right)-z\left(s, b, \nu^{0}\right)\right) \mid P^{0}(d a d b) d s \\
& \left.+\|K\|_{L i p} \int_{0}^{t} \iiint \int \mid z\left(s, a^{\prime}, \mu^{0}\right)-z\left(s, b^{\prime}, \nu^{0}\right)\right) \mid P^{0}\left(d a^{\prime} d b^{\prime}\right) P^{0}(d a d b) d s
\end{aligned}
$$

which implies that

$$
\begin{gathered}
\iint\left|z\left(t, a, \mu^{0}\right)-z\left(t, b, \nu^{0}\right)\right| P^{0}(d a d b) \leq \iint|a-b| P^{0}(d a d b) \\
\left.+2\|K\|_{\text {Lip }} \int_{0}^{t} \iint \mid z\left(s, a, \mu^{0}\right)-z\left(s, b, \nu^{0}\right)\right) \mid P^{0}(d a d b) d s
\end{gathered}
$$

or in other words

$$
\Phi(t) \leq \Phi(0)+2\|K\|_{\text {Lip }} \int_{0}^{t} \Phi(s) d s .
$$

By Gronwall's inequality

$$
\Phi(t) \leq \Phi(0) e^{2 t\|K\|_{L i p}}
$$

and taking the infimum of both sides of this inequality over $E\left(\mu^{0}, \nu^{0}\right)$ leads to Dobrushin's inequality.

At this point, Dobrushin's inequality can be used in two different ways in the proof of the mean-field limit:

- it proves in particular the uniqueness of the solution to (I.7) within the class $C\left(\mathbf{R}_{+}, w^{*}-\mathcal{M}^{1}\left(\mathbf{R}^{D}\right)\right)$

- if one takes $\nu^{0, N}$ to be of the form

$$
\nu^{0, N}=\frac{1}{N} \sum_{k=1}^{N} \delta_{z_{k}^{0, N}},
$$

then for each $k \geq 1, z_{k}^{N}\left(t, z_{k}^{0, N}, \nu^{0, N}\right)$ is the solution to the system of ODE

$$
\begin{aligned}
z_{k}^{N}\left(t, z_{k}^{0, N}, \nu^{0, N}\right) & =\frac{1}{N} \sum_{l=1}^{N} K\left(z_{k}^{N}\left(t, z_{k}^{0, N}, \nu^{0, N}\right)-z_{l}^{N}\left(t, z_{l}^{0, N}, \nu^{0, N}\right)\right) \\
z_{k}^{N}\left(0, z_{k}^{0, N}, \nu^{0, N}\right) & =z_{k}^{0, N}
\end{aligned}
$$

then, by setting

$$
\nu^{N}(t)=\frac{1}{N} \sum_{k=1}^{N} \delta_{z_{k}^{N}\left(t, z_{k}^{0, N}, \nu^{0, N}\right)},
$$

a direct application of Dobrushin's inequality shows that

$$
W\left(\mu(t), \nu^{N}(t)\right) \rightarrow 0 \text { for each } t>0 \text { as } N \rightarrow+\infty
$$

if $W\left(\mu^{0}, \nu^{0, N}\right) \rightarrow 0$. Since the Wasserstein distance metricizes the weak-* topology of $\mathcal{M}^{1}\left(\mathbf{R}^{D}\right)$, this gives another proof of the mean-field limit as in Theorem I.6 


\section{I.B. Chaotic sequences}

In Theorem I.6, we proved the convergence of the empirical distribution of particles to the solution of Vlasov's equation in the large $N$ limit and for the weak coupling scaling.

Even if the empirical distribution is a natural object encoding the motion of $N$ particles under the Hamiltonian flow of (I.1), it is a probability measure on the 1-particle phase space. Another, equally natural object to consider is the joint distribution of the same system of $N$ undistinguishable particles which is a probability measure on the $N$-particle phase space.

The next Lemma explains how both objects are related.

More generally, this Appendix is aimed at explaining in detail the respective roles of the $N$-particle and the 1-particle phase spaces in the mean-field limit.

Lemma I.8. Let $f \in \mathcal{M}^{1}\left(\mathbf{R}^{D}\right)$ and, for all $N \geq 1, F_{N} \in \mathcal{M}^{1}\left(\left(\mathbf{R}^{D}\right)^{N}\right)$ symmetric in all variables - i.e. $F_{N}$ is invariant under the transformation

$$
\left(x_{1}, \ldots, x_{N}\right) \mapsto\left(x_{\sigma(1)}, \ldots, x_{\sigma(N)}\right)
$$

for each $\sigma \in \mathfrak{S}_{N}$. The two following statements are equivalent (1) for each $\epsilon>0$ and each $\phi \in C_{c}\left(\mathbf{R}^{D}\right)$,

$$
F_{N}\left(\left\{\left(x_{1}, \ldots, x_{N}\right) \in\left(\mathbf{R}^{D}\right)^{N}||\left\langle\frac{1}{N} \sum_{k=1}^{N} \delta_{x_{k}}-f, \phi\right\rangle \mid \geq \epsilon\right\}\right) \rightarrow 0
$$

as $N \rightarrow+\infty$;

(2) the sequence $F_{N: j}$ of marginal distributions of $F_{N}$ - i.e. $F_{N: j}$ is the image of $F_{N}$ under the projection $\left(x_{1}, \ldots, x_{N}\right) \mapsto\left(x_{1}, \ldots, x_{j}\right)$ - satisfies

$$
F_{N: j} \rightarrow f^{\otimes j} \text { weakly-* }^{*}, \quad \text { as } N \rightarrow+\infty .
$$

Proof. Let us show how (1) implies (2). First, (1) clearly implies that

$$
\mathbf{E}^{F_{N}}\left|\left\langle\frac{1}{N} \sum_{k=1}^{N} \delta_{x_{k}}-f, \phi\right\rangle\right|^{j} \rightarrow 0 \text { for all } j \geq 1
$$

as $N \rightarrow+\infty$. For $j=1$, observe that, by symmetry of $F_{N}$,

$$
\begin{aligned}
\mathbf{E}^{F_{N}}\left\langle\frac{1}{N} \sum_{k=1}^{N} \delta_{x_{k}}-f, \phi\right\rangle & =\mathbf{E}^{F_{N}}\left(\frac{1}{N} \sum_{k=1}^{N} \phi\left(x_{k}\right)\right)-\langle f, \phi\rangle \\
& =\mathbf{E}^{F_{N: 1}} \phi-\langle f, \phi\rangle
\end{aligned}
$$

and hence

$$
F_{N: 1} \rightarrow f \text { weakly- }^{*} \text { as } N \rightarrow+\infty
$$

For $j=2$

$$
\begin{aligned}
\mathbf{E}^{F_{N}}\left(\left\langle\frac{1}{N} \sum_{k=1}^{N} \delta_{x_{k}}-f, \phi\right\rangle\right)^{2} & =\mathbf{E}^{F_{N}}\left(\frac{1}{N} \sum_{k=1}^{N} \phi\left(x_{k}\right)\right)^{2} \\
& -2\langle f, \phi\rangle \mathbf{E}^{F_{N}}\left(\frac{1}{N} \sum_{k=1}^{N} \phi\left(x_{k}\right)\right)+\langle f, \phi\rangle^{2} .
\end{aligned}
$$


On the other hand

$$
\begin{aligned}
& \mathbf{E}^{F_{N}}\left(\frac{1}{N} \sum_{k=1}^{N} \phi\left(x_{k}\right)\right)^{2}=\frac{1}{N^{2}} \mathbf{E}^{F_{N}} \sum_{k=1}^{N} \phi\left(x_{k}\right)^{2} \\
& +\frac{1}{N^{2}} \mathbf{E}^{F_{N}} \sum_{1 \leq k \neq l \leq N} \phi\left(x_{k}\right) \phi\left(x_{l}\right) \\
& =\frac{1}{N} \mathbf{E}^{F_{N: 1}} \phi^{2}+\frac{N(N-1)}{N^{2}} \mathbf{E}^{F_{N: 2}} \phi^{\otimes 2}
\end{aligned}
$$

so that, by using again the symmetry of $F_{N}$,

$$
\begin{array}{r}
\mathbf{E}^{F_{N}}\left(\left\langle\frac{1}{N} \sum_{k=1}^{N} \delta_{x_{k}}-f, \phi\right\rangle\right)^{2}=\frac{N(N-1)}{N^{2}} \mathbf{E}^{F_{N: 2}} \phi^{\otimes 2} \\
-2\langle f, \phi\rangle \mathbf{E}^{F_{N: 1}} \phi+\langle f, \phi\rangle^{2}+\frac{1}{N} \mathbf{E}^{F_{N: 1}} \phi^{2}
\end{array}
$$

Hence, if

$$
\mathbf{E}^{F_{N}}\left(\left\langle\frac{1}{N} \sum_{k=1}^{N} \delta_{x_{k}}-f, \phi\right\rangle\right)^{2} \rightarrow 0
$$

as $N \rightarrow+\infty$, one has

$$
F_{N: 2} \rightarrow f^{\otimes 2} \text { weakly-* as } N \rightarrow+\infty .
$$

The extension to $j$ s larger than 2 is done in the same manner.

Conversely, assume that (2) holds for $j=1,2$. By the Bienayme-Chebyshev inequality

$$
\begin{aligned}
& F_{N}\left(\left\{\left(x_{1}, \ldots, x_{N}\right) \in\left(\mathbf{R}^{D}\right)^{N}\right.\right.\left.\left.\left|\left\langle\frac{1}{N} \sum_{k=1}^{N} \delta_{x_{k}}-f, \phi\right\rangle\right| \geq \epsilon\right\}\right) \\
& \leq \frac{1}{\epsilon^{2}} \mathbf{E}^{F_{N}}\left\langle\frac{1}{N} \sum_{k=1}^{N} \delta_{x_{k}}-f, \phi\right\rangle^{2}
\end{aligned}
$$

so that, because of (I.10)

$$
\begin{aligned}
& F_{N}\left(\left\{\left(x_{1}, \ldots, x_{N}\right) \in\left(\mathbf{R}^{D}\right)^{N}||\left\langle\frac{1}{N} \sum_{k=1}^{N} \delta_{x_{k}}-f, \phi\right\rangle \mid \geq \epsilon\right\}\right) \\
& =\frac{1}{\epsilon^{2}}\left(\frac{N(N-1)}{N^{2}} \mathbf{E}^{\left.F_{N: 2} \phi^{\otimes 2}-2\langle f, \phi\rangle \mathbf{E}^{F_{N: 1}} \phi+\langle f, \phi\rangle^{2}+\frac{1}{N} \mathbf{E}^{F_{N: 1}} \phi^{2}\right) .}\right.
\end{aligned}
$$

Keeping $\epsilon>0$ fixed and letting $N \rightarrow+\infty$ leads to (1) because of the limits

$$
\mathbf{E}^{F_{N: 1}} \phi \rightarrow\langle f, \phi\rangle, \quad \mathbf{E}^{F_{N: 2}} \phi^{\otimes 2} \rightarrow\langle f, \phi\rangle^{2}
$$

as $N \rightarrow+\infty$. 
Definition I.9. A sequence $F_{N} \in \mathcal{M}^{1}\left(X^{N}\right)$ that satisfies either one of the equivalent statements in Lemma I.8 is called "chaotic".

An obvious example of chaotic sequence is $F_{N}=f^{\otimes N}$. In this case, and for $X=\mathbf{R}^{D}$, the condition (1) in Lemma I.8 can be improved as follows.

Define $\Omega=\left(\mathbf{R}^{D}\right)^{\mathbf{N}^{*}}$ to be the space of $\mathbf{R}^{D}$-valued sequences $\left(a_{n}\right)_{n \geq 1}$, endowed with the $\sigma$-algebra generated by cylinders ${ }^{4}$. The infinite tensor product $F_{\infty}=f \mathbf{N}^{*}$ defines a probability measure on $\Omega$ with that $\sigma$-algebra.

Lemma I.10. For $F_{\infty}$-a.e. $a=\left(a_{1}, a_{2}, a_{3}, \ldots\right) \in \Omega$, the empirical measure

$$
f_{N, a}=\frac{1}{N} \sum_{k=1}^{N} \delta_{a_{k}} \rightarrow f \text { weakly-* on } \mathbf{R}^{D} .
$$

In the particular case of $F_{\infty}=f^{\otimes \mathbf{N}^{*}}$ considered here, condition (1) in Lemma I. 8 can be recast as

$$
\lim _{N \rightarrow+\infty} F_{\infty}\left(\left\{a \in \Omega||\left\langle f_{N, a}-f, \phi\right\rangle \mid>\epsilon\right\}\right)=0
$$

for each $\epsilon>0$; in other words, it expresses that, for all $\phi \in C_{c}\left(\mathbf{R}^{D}\right)$

$$
\left\langle f_{N, a}, \phi\right\rangle \rightarrow\langle f, \phi\rangle \text { in } F_{\infty} \text {-probability as } N \rightarrow+\infty,
$$

while Lemma I.10 says that

$$
\left\langle f_{N, a}, \phi\right\rangle \rightarrow\langle f, \phi\rangle F_{\infty} \text {-a.e. as } N \rightarrow+\infty,
$$

which is a stronger statement (we recall that a.e.- convergence is a stronger notion of convergence than convergence in probability - more precisely, a.e.-convergence implies convergence in probability, while convergence in probability implies only the a.e.-convergence modulo extraction of subsequences).

Proof. Pick $\phi \in C_{c}\left(\mathbf{R}^{D}\right)$ and set $Y_{n}(a)=\phi\left(a_{n}\right)$. Clearly, the random variables $\left(Y_{n}\right)_{n \geq 1}$ are independent on $\Omega$ and identically distributed (under $f$ ). By the strong law of large numbers,

$$
\frac{1}{N} \sum_{n=1}^{N} Y_{n}(a) \rightarrow \mathbf{E}^{F_{\infty}}\left(Y_{n}\right)=\langle f, \phi\rangle F_{\infty} \text {-a.e. on } \Omega \text { as } N \rightarrow+\infty \text {. }
$$

By a classical separability argument, one can choose the $F_{\infty}$-negligible set in the statement above to be independent of $\phi$, which gives the announced statement.

Let us go back to our original problem, namely the mean-field limit of a system of $N$ particles. Our aim is to apply the notion of chaotic sequence to that problem. We shall do so for the first-order mean-field PDE on which we explained Dobrushin's

\footnotetext{
${ }^{4}$ i.e. sets of the form $\prod_{n \geq 1} B_{n}$, with $B_{n}$ a Borel set in $\mathbf{R}^{D}$ for each $n \geq 1$ and $B_{n}=\mathbf{R}^{D}$ for all but a finite number of $n \mathrm{~s}$.
} 
inequality, rather than on the Vlasov equation itself, the extension of arguments to this latter case being obvious.

Given $Z_{N}^{0}=\left(z_{1}^{0}, \ldots, z_{N}^{0}\right)$, let $Z_{N}\left(t, Z_{N}^{0}\right)=\left(z_{1}\left(t, Z_{N}^{0}\right), \ldots, z_{N}\left(t, Z_{N}^{0}\right)\right)$ be the solution of the system of ODEs

$$
\begin{aligned}
& \dot{z}_{k}\left(t, Z_{N}^{0}\right)=\frac{1}{N} \sum_{l=1}^{N} K\left(z_{k}\left(t, Z_{N}^{0}\right)-z_{l}\left(t, Z_{N}^{0}\right)\right) \\
& z_{k}\left(0, Z_{N}^{0}\right)=z_{k}^{0}, \quad k=1, \ldots, N .
\end{aligned}
$$

We designate by $f_{N, Z_{N}^{0}}(t)$ the empirical measure

$$
f_{N, Z_{N}^{0}}(t)=\frac{1}{N} \sum_{k=1}^{N} \delta_{z_{k}\left(t, Z_{N}^{0}\right)} .
$$

Given $f^{0} \in \mathcal{M}^{1}\left(\mathbf{R}^{D}\right)$, we denote by $F_{N}(t)$ the image of $\left(f^{0}\right)^{\otimes N}$ under the map $Z_{N}^{0} \mapsto Z_{N}\left(t, Z_{N}^{0}\right)$, i.e.

$$
F_{N}(t)=Z_{N}(t, \cdot)_{*}\left(f^{0}\right)^{\otimes N} .
$$

Theorem I.11. Assume that $K$ is Lipschitz continuous on $\mathbf{R}^{D}$, and let $f^{0} \in$ $\mathcal{M}^{1}\left(\mathbf{R}^{D}\right)$. Then, for each $n \geq 1$ and each $t>0$,

$$
F_{N: n}(t) \rightarrow f(t)^{\otimes n} \text { weak- }^{*} \text { as } N \rightarrow+\infty
$$

where $f(t)$ is the solution to the mean-field PDE

$$
\partial_{t} f+\operatorname{div}_{z}\left(\left(K \star_{z} f\right) f\right)=0,\left.\quad f\right|_{t=0}=f^{0} .
$$

Proof. Let $h \in C_{c}\left(\left(\mathbf{R}^{D}\right)^{m}\right)$; one has

$$
\mathbf{E}^{F_{N}(t)}\left\langle\left(f_{N, Z_{N}^{0}}^{0}\right)^{\otimes m}, h\right\rangle=\mathbf{E}^{F_{N}(0)}\left\langle\left(f_{N, Z_{N}^{0}}(t)\right)^{\otimes m}, h\right\rangle=\mathbf{E}^{F_{\infty}}\left\langle\left(f_{N, \bullet}(t)\right)^{\otimes m}, h\right\rangle
$$

in the notation of Lemma I.10.

For $F_{\infty}$-a.e. $a \in \Omega$, Lemma I.10 implies that

$$
f_{N, a}(0) \rightarrow f^{0} \text { weakly- }^{*} \text { as } N \rightarrow+\infty .
$$

By the analogue of Theorem I.6 adapted to the mean-field limit of (I.11) which follows directly from Dobrushin's inequality - one has

$$
f_{N, a}(t) \rightarrow f(t) \text { weakly-* as } N \rightarrow+\infty
$$

for $F_{\infty}$-a.e. $a \in \Omega$ and each $t>0$.

Hence, given any $h \in C_{c}\left(\left(\mathbf{R}^{D}\right)^{m}\right)$,

$$
\left\langle\left(f_{N, a}(t)\right)^{\otimes m}, h\right\rangle \rightarrow\left\langle f(t)^{\otimes m}, h\right\rangle \text { weakly-* as } N \rightarrow+\infty
$$

for $F_{\infty}$-a.e. $a \in \Omega$ and each $t>0$. On the other hand, one has

$$
\left|\left\langle\left(f_{N, a}(t)\right)^{\otimes m}, h\right\rangle\right| \leq\|h\|_{L^{\infty}}
$$


so that, by dominated convergence

$$
\mathbf{E}^{F_{N}(t)}\left\langle\left(f_{N, Z_{N}^{0}}^{0}\right)^{\otimes m}, h\right\rangle=\mathbf{E}^{F_{\infty}}\left\langle\left(f_{N, \bullet}(t)\right)^{\otimes m}, h\right\rangle \rightarrow\left\langle f(t)^{\otimes m}, h\right\rangle
$$

as $N \rightarrow+\infty$, for each $t>0$.

Using the Bienayme-Chebyshev inequality as in the proof of Lemma I.8 shows that, for each $t>0, F_{N}(t)$ satisfies condition (1) in that Lemma. Hence it must also satisfy condition (2) there, which is precisely our claim.

In other words, if $F_{N}(t)$ is the solution to the Liouville equation

$$
\begin{gathered}
\partial_{t} F_{N}+\sum_{1 \leq k, l \leq N} \operatorname{div}_{z_{k}}\left(K\left(z_{k}-z_{l}\right) F_{N}\right)=0, \\
F_{N}(0)=\left(f^{0}\right)^{\otimes N},
\end{gathered}
$$

with $f^{0} \in \mathcal{M}^{1}\left(\mathbf{R}^{D}\right)$, then, for each $t>0, F_{N}(t)$ is a chaotic sequence and its marginal distributions satisfy

$$
F_{N: n}(t) \rightarrow f(t)^{\otimes n} \text { weakly-* as } N \rightarrow+\infty
$$

for each $t>0$, where $f$ is the solution to (I.12). Thus, the fact that $F_{N}(0)=\left(f^{0}\right)^{\otimes N}$, i.e. that $F_{N}(0)$ is the simplest chaotic sequence imaginable, implies that $F_{N}(t)$ is chaotic for all $t>0$. This property is called propagation of chaos and is of paramount importance in Statistical Mechanics.

\section{Part II. From the quantum $N$-body problem to Hartree's equation}

In this section, we explain how the nonlinear, mean-field Schrödinger equation (1.6) can be derived from the linear $N$-body Schrödinger equation (1.20). One way of doing this is through the weak-coupling scaling similar to (I.2) in the classical case. Hence the potential $V$ is replaced by $V / N$ in (1.19), leading to

$$
\mathcal{H}_{N}=\sum_{k=1}^{N}-\frac{1}{2} \Delta_{x_{k}}+\frac{1}{N} \sum_{1 \leq k<l \leq N} V\left(x_{k}, x_{l}\right) .
$$

(Here, for simplicity, we switch to dimensionless variables, so that $\hbar=m=1$ ). We assume that the potential $V$ in (II.1) satisfies

$$
V \in L^{\infty}\left(\mathbf{R}^{D} \times \mathbf{R}^{D}\right), \quad V(x, y)=V(y, x) \in \mathbf{R} \text { for a.e. } x, y \in \mathbf{R}^{D} .
$$

The quantum, $N$-body Hamiltonian $\mathcal{H}_{N}$ in (II.1) is an unbounded self-adjoint operator on $L^{2}\left(\left(\mathbf{R}^{D}\right)^{N}\right)$ with domain $H^{2}\left(\left(\mathbf{R}^{D}\right)^{N}\right)$. By Stone's theorem, $-\frac{i}{\hbar} \mathcal{H}_{N}$ generates a unitary group on $L^{2}\left(\left(\mathbf{R}^{D}\right)^{N}\right)$. Thus, given any $\Psi_{N}^{0} \in H^{2}\left(\left(\mathbf{R}^{D}\right)^{N}\right)$, e $e^{-i t \mathcal{H}_{N} / \hbar} \Psi_{N}^{0}$ defines the only classical solution $\Psi_{N}$ to the linear $N$-body Schrödinger equation

$$
\begin{aligned}
i \partial_{t} \Psi_{N}=\mathcal{H}_{N} \Psi_{N} & =-\frac{1}{2} \sum_{k=1}^{N} \Delta_{x_{k}} \Psi_{N} \\
& +\frac{1}{N} \sum_{1 \leq k<l \leq N} V\left(x_{k}-x_{l}\right) \Psi_{N}\left(t, X_{N}, Y_{N}\right) \\
\left.\Psi_{N}\right|_{t=0} & =\Psi_{N}^{0} .
\end{aligned}
$$




\section{II.1. The quantum BBGKY hierarchy}

We shall work with density operators rather than with wave functions. If $\Psi_{N}$ solves the $N$-body Schrödinger equation (II.3), then the density operator

$$
D_{N}(t):=\left|\Psi_{N}(t, \cdot)\right\rangle\left\langle\Psi_{N}(t, \cdot)\right|
$$

- i.e. the integral operator on on $L^{2}\left(\left(\mathbf{R}^{D}\right)^{N}\right)$ with integral kernel

$$
D_{N}\left(t, X_{n}, Y_{N}\right)=\Psi_{N}\left(t, X_{N}\right) \overline{\Psi_{N}\left(t, Y_{N}\right)}
$$

- solves the $N$-body von Neumann equation

$$
\begin{aligned}
i \partial_{t} D_{N} & =\left[\mathcal{H}_{N}, D_{N}\right], \\
\left.D_{N}\right|_{t=0} & =\left|\Psi_{N}^{0}\right\rangle\left\langle\Psi_{N}^{0}\right| .
\end{aligned}
$$

In terms of the density matrix, this equation becomes

$$
\begin{aligned}
i \partial_{t} D_{N} & =-\frac{1}{2} \sum_{k=1}^{N}\left(\Delta_{x_{k}}-\Delta_{y_{k}}\right) D_{N} \\
& +\frac{1}{N} \sum_{1 \leq k<l \leq N}\left(V\left(x_{k}-x_{l}\right)-V\left(y_{k}-y_{l}\right)\right) D_{N}\left(t, X_{N}, Y_{N}\right) \\
\left.D_{N}\right|_{t=0} & =\Psi_{N}^{0}\left(X_{N}\right) \overline{\Psi_{N}^{0}\left(Y_{N}\right)} .
\end{aligned}
$$

(Throughout this course, we designate by the same letter the integral operator $D_{N}(t)$ — the density operator - and its integral kernel — the density matrix).

Below, we assume that the initial data is factorized, i.e. that

$$
\Psi_{N}^{0}\left(x_{1}, \ldots, x_{N}\right)=\prod_{k=1}^{N} \psi^{0}\left(x_{k}\right)
$$

where $\psi^{0} \in H^{2}\left(\mathbf{R}^{D}\right)$. Because of the interaction modelled by the binary potential $V, \Psi_{N}(t, \cdot)$ is usually not factorized for $t>0$.

However, the symmetry

$$
\Psi_{N}^{0}\left(x_{1}, \ldots, x_{N}\right)=\Psi_{N}^{0}\left(x_{\sigma(1)}, \ldots, x_{\sigma(N)}\right), \quad \sigma \in \mathfrak{S}_{N}
$$

is obviously propagated by $e^{-i t \mathcal{H}_{N} / \hbar}$ :

$$
\Psi_{N}\left(t, x_{1}, \ldots, x_{N}\right)=\Psi_{N}\left(t, x_{\sigma(1)}, \ldots, x_{\sigma(N)}\right), \quad t>0, \sigma \in \mathfrak{S}_{N}
$$

At this point, we must depart from the strategy used for the limit that leads from the classical $N$-body problem to Vlasov's equation. Indeed, there is no quantum analogue of the empirical distribution — or equivalently, whatever quantum analogue of the empirical distribution one may think of is in general not a solution to Hartree's equation

$$
\begin{aligned}
i \partial_{t} \psi(t, x)+\frac{1}{2} \Delta_{x} \psi(t, x) & =\psi(t, x) \int V(x-y)|\psi(t, y)|^{2} d y \\
\psi(0, x) & =\psi^{0}(x)
\end{aligned}
$$


Thus, Hartree's approximation is not a statement on the continuous dependence upon initial data of the solution to Hartree's equation.

Fortunately, the discussion in Appendix B, and especially Lemma I.8 there suggests another strategy, namely to study the sequence of marginal distributions coming from the $N$-particle density. In the quantum case, one must consider density matrices - i.e. operators - instead of functions.

For $n=1, \ldots, N$, define $D_{N: n}(t)$, the $n$-th reduced density matrix of $D_{N}$, by the formula

$$
D_{N: n}\left(t, X_{n}, Y_{n}\right)=\int D_{N}\left(t, X_{n}, Z_{n}^{N}, Y_{n}, Z_{n}^{N}\right) d Z_{n}^{N}
$$

with the notation

$$
Z_{n}^{N}=\left(z_{n+1}, \ldots, z_{N}\right), \quad n<N
$$

Since the sequence of $D_{N: n}$ 's are the objects of interest, it is natural to seek an equation - or more exactly a system of equations - governing their evolution. This is done in the following manner: reducing the $N-n$ variables to the diagonal and integrating them out, one finds that

$$
\begin{aligned}
& i \partial_{t} D_{N: n}\left(t, X_{n}, Y_{n}\right)+\frac{1}{2} \sum_{k=1}^{n}\left(\Delta_{x_{k}}-\Delta_{y_{k}}\right) D_{N: n}\left(t, X_{n}, Y_{n}\right) \\
& =\frac{1}{N} \sum_{1 \leq k<l \leq n}\left(V\left(x_{k}-x_{l}\right)-V\left(y_{k}-y_{l}\right)\right) D_{N: n}\left(t, X_{n}, Y_{n}\right) \\
& +\frac{1}{N} \sum_{\substack{1 \leq k \leq n \\
n+1 \leq l \leq N}} \int\left(V\left(x_{k}-z_{l}\right)-V\left(y_{k}-z_{l}\right)\right) D_{N}\left(t, X_{n}, Z_{n}^{N}, Y_{n}, Z_{n}^{N}\right) d Z_{n}^{N} \\
& +\frac{1}{N} \sum_{n+1 \leq k<l \leq N} \int\left(V\left(z_{k}-z_{l}\right)-V\left(z_{k}-z_{l}\right)\right) D_{N}\left(t, X_{n}, Z_{n}^{N}, Y_{n}, Z_{n}^{N}\right) d Z_{n}^{N}
\end{aligned}
$$

The last term on the right-hand side of the above equality is obviously 0 ; the second one can be simplified in the following manner. Observe that, by the symmetry (II.8), one has

$$
\begin{aligned}
D_{N}\left(t, x_{\sigma(1)}, \ldots, x_{\sigma(N)}, y_{\sigma(1)}, \ldots,\right. & \left.y_{\sigma(N)}\right)=D_{N}\left(t, X_{N}, Y_{N}\right) \\
& \text { for all } \sigma \in \mathfrak{S}_{N}, X_{N}, Y_{N} \in\left(\mathbf{R}^{D}\right)^{N} .
\end{aligned}
$$

Hence, if $l \neq n+1$, in the integral

$$
\int\left(V\left(x_{k}-z_{l}\right)-V\left(y_{k}-z_{l}\right)\right) D_{N}\left(t, X_{n}, Z_{n}^{N}, Y_{n}, Z_{n}^{N}\right) d Z_{n}^{N}
$$

we change variables by $\left(z_{l}, z_{n+1}\right) \mapsto\left(z_{n+1}, z_{l}\right)$, leaving all the other variables unchanged. Because of the symmetry (II.12), this does not change the density matrix, so that the above integral satisfies

$$
\begin{aligned}
\int & \left(V\left(x_{k}-z_{l}\right)-V\left(y_{k}-z_{l}\right)\right) D_{N}\left(t, X_{n}, Z_{n}^{N}, Y_{n}, Z_{n}^{N}\right) d Z_{n}^{N} \\
& =\int\left(V\left(x_{k}-z_{n+1}\right)-V\left(y_{k}-z_{n+1}\right)\right) D_{N}\left(t, X_{n}, Z_{n}^{N}, Y_{n}, Z_{n}^{N}\right) d Z_{n}^{N} \\
& =\int\left(V\left(x_{k}-z_{n+1}\right)-V\left(y_{k}-z_{n+1}\right)\right) D_{N: n+1}\left(t, X_{n}, z_{n+1}, Y_{n}, z_{n+1}\right) d z_{n+1}
\end{aligned}
$$


after integrating over $Z_{n+1}^{N}$. Hence the system of equations that govern the reduced density matrices $D_{N: n}$ is

$$
\begin{aligned}
& i \partial_{t} D_{N: n}\left(t, X_{n}, Y_{n}\right)+\frac{1}{2} \sum_{k=1}^{n}\left(\Delta_{x_{k}}-\Delta_{y_{k}}\right) D_{N: n}\left(t, X_{n}, Y_{n}\right) \\
& \quad=\frac{N-n}{N} \sum_{k=1}^{n} \int\left(V\left(x_{k}-z\right)-V\left(y_{k}-z\right)\right) D_{N: n+1}\left(t, X_{n}, z, Y_{n}, z\right) d z \\
& +\frac{1}{N} \sum_{1 \leq k<l \leq n}\left(V\left(x_{k}-x_{l}\right)-V\left(y_{k}-y_{l}\right)\right) D_{N: n}\left(t, X_{n}, Y_{n}\right), \quad n \geq 1
\end{aligned}
$$

with the convention

$$
D_{N: N}=D_{N}, \quad D_{N: n} \equiv 0 \text { for all } n>N
$$

This system of equations is known as the "Bogolyubov-Born-Green-Kirkwood-Yvon" (abbreviated as BBGKY) hierarchy".

Notice that the $N$-th equation in this hierarchy is nothing but the $N$-body von Neumann equation (II.6); hence the $N-1$ first equations in the BBGKY hierarchy contain no additional information. This observation might cast doubts on the relevance of the BBGKY hierarchy.

However, here is how this hierarchy is used to establish the mean-field limit. Keeping $n$ fixed, we let $N \rightarrow+\infty$ in the $n$-th equation of the BBGKY hierarchy, assuming that

$$
D_{N: n}\left(t, X_{n}, Y_{n}\right) \rightarrow D_{n}\left(t, X_{n}, Y_{n}\right) \text { as } N \rightarrow+\infty
$$

in some sense that will be described later. Taking limits in (II.13), we arrive at the infinite BBGKY hierarchy

$$
\begin{aligned}
& i \partial_{t} D_{n}\left(t, X_{n}, Y_{n}\right)+\frac{1}{2} \sum_{k=1}^{n}\left(\Delta_{x_{k}}-\Delta_{y_{k}}\right) D_{n}\left(t, X_{n}, Y_{n}\right) \\
& \quad=\sum_{k=1}^{n} \int\left(V\left(x_{k}-z\right)-V\left(y_{k}-z\right)\right) D_{n+1}\left(t, X_{n}, z, Y_{n}, z\right) d z, \quad n \geq 1
\end{aligned}
$$

since the second term on the right-hand side of (II.13) is (formally) of order $O\left(n^{2} / N\right)$, while $\frac{N-n}{N} \rightarrow 1$ as $N \rightarrow+\infty$, so that the first term on the right-hand side of (II.13) is (formally) of order $n$. Because of the assumption (II.7) on the initial data, one has

$$
D_{n}\left(0, X_{n}, Y_{n}\right)=\prod_{k=1}^{n} \psi^{0}\left(x_{k}\right) \overline{\psi^{0}\left(y_{k}\right)} .
$$

In this infinite hierarchy, there is no particular equation that entails all the other, as is the case of the finite BBGKY hierarchy.

However, here is a fundamental property of the infinite hierarchy. 
Proposition II.1. Let $\psi$ be the solution to Hartree's equation (II.9) with $\left\|\psi^{0}\right\|_{L^{2}}=$ 1. Then the sequence of density matrices

$$
F_{n}\left(t, X_{n}, Y_{n}\right)=\prod_{k=1}^{n} \psi(t, x) \overline{\psi(t, y)}
$$

is a solution to the infinite BBGKY hierarchy (II.15) with initial data (II.16).

The proof of this Proposition is done by inspection. Observe that the sequence of functions (II.17) is not a solution to the BBGKY hierarchy (II.13) for finite $N$ : in other words, factorization appears in the limit as $N \rightarrow+\infty$ only.

Since one can expect to prove that the sequence of solutions to the BBGKY hierarchy (II.13) converges to solutions of (II.15), the observation above suggests the natural question: is the factorized solution (II.17) the unique solution to (II.15) with initial data (II.16)?

\section{II.2. Handling the BBGKY hierarchy with the Cauchy-Kova- levski theorem}

It is of paramount importance for the rest of the discussion to have a clear picture of this notion of infinite hierarchy of equations - and especially of its meaning.

Start from the simplest nonlinear ODE - Riccati equation - in the form

$$
\dot{y}=y^{2}, \quad y(0)=y^{i n},
$$

whose solution is

$$
y(t, x)=\frac{y^{i n}}{1-t y^{i n}}, \quad t<\frac{1}{y^{i n}} .
$$

We embed this nonlinear ODE into an infinite hierarchy of linear ODEs by the prescription

$$
x_{n}(t)=y(t)^{n}, \quad n \geq 1,
$$

which leads to

$$
\dot{x}_{n}(t)=n x_{n+1}(t), \quad x_{n}(0)=\left(y^{i n}\right)^{n}, \quad n \geq 1 .
$$

The simple form of this hierarchy suggests considering the generating function

$$
f(t, z)=\sum_{n \geq 1} x_{n+1}(t) z^{n}
$$

As long as $z$ remains in the open disk of convergence and $x_{n}$ is differentiable at $t$, one has

$$
\partial_{t} f(t, z)=\sum_{n \geq 0}(n+1) x_{n+2}(t) z^{n}=\partial_{z}\left(\sum_{n \geq 0} x_{n+2}(t) z^{n+1}\right)=\partial_{z} f(t, z),
$$

while

$$
f(0, z)=\sum_{n \geq 0}\left(y^{i n}\right)^{n+1} z^{n}=\frac{y^{i n}}{1-z y^{i n}} .
$$


Hence

$$
f(t, z)=f(0, z+t)=\frac{y^{i n}}{1-(z+t) y^{i n}} .
$$

In particular, the solution $y(t)$ to Riccati's equation (II.18) is found as

$$
y(t)=x_{1}(t)=f(t, 0)=\frac{y^{i n}}{1-(z+t) y^{\text {in }}} .
$$

This is certainly the most difficult way to solve (II.18), yet this discussion suggests that the interaction term in $n$-th equation of the infinite hierarchy (II.15), namely

$$
\sum_{k=1}^{n} \int\left(V\left(x_{k}-z\right)-V\left(y_{k}-z\right)\right) D_{n+1}\left(t, X_{n}, z, Y_{n}, z\right) d z
$$

should be regarded as a first-order differential operator acting on the string of unknowns $D_{n}$.

The appropriate mathematical tool to do this is the following abstract variant of the Cauchy-Kovalevski theorem, due to Nirenberg [27] and Nishida [29]

Theorem II.2. Let $B_{\rho}\left(0<\rho<\rho_{0}\right)$ be a decreasing scale of Banach spaces, and let $F(t, u)$ be a continuous mapping defined for $u \in B_{\rho},\|u\|_{\rho} \leq R,|t|<\eta(R>0, \eta>0$ are fixed) with values in $B_{\rho^{\prime}}$ for every $\rho, \rho^{\prime}$ such that $0<\rho^{\prime}<\rho<\rho_{0}$. Assume that

(1) $\|F(u, t)-F(v, t)\|_{\rho^{\prime}} \leq C\|u-v\|_{\rho} /\left(\rho-\rho^{\prime}\right)$;

(2) $\|F(0, t)\|_{\rho} \leq$ const $/\left(\rho_{0}-\rho\right)$.

Then there exists a positive constant a such that the problem

$$
\begin{aligned}
& \dot{u}(t)=F(t, u(t)), \quad|t|<a\left(\rho_{0}-\rho\right) \\
& u(0)=0
\end{aligned}
$$

has a unique solution $u \in C\left(\left[-a\left(\rho_{0}-\rho\right), a\left(\rho_{0}-\rho\right)\right], B_{\rho}\right)$, for every $\rho$ such that $0<\rho<\rho_{0}$.

We refer to Nishida's original article ([29]) for an elegant proof of this result.

Next we explain how to apply this Cauchy-Kovalevski theorem to an infinite hierarchy as (II.15). We do so by considering an abstract variant of (II.15), as follows:

$$
\begin{aligned}
\dot{u}_{n}(t) & =\mathcal{A}_{n} u_{n}(t)+\mathcal{C}_{n, n+1} u_{n+1}(t), \quad n \geq 1, \\
u_{n}(0) & =u_{n}^{i n}
\end{aligned}
$$

Here $\mathcal{A}_{n}$ is an unbounded operator on a Banach space $\mathcal{E}_{n}$ - whose norm is denoted by $\|\cdot\|_{n}$ - and generates a group of isometries on $\mathcal{E}_{n}$ denoted by $\mathcal{S}_{n}(t)$. On the other hand, for each $n \geq 1$, one has $\mathcal{C}_{n, n+1} \in \mathcal{L}\left(\mathcal{E}_{n+1}, \mathcal{E}_{n}\right)$ with

$$
\left\|\mathcal{C}_{n, n+1}\right\|_{\mathcal{L}\left(\mathcal{E}_{n+1}, \mathcal{E}_{n}\right)} \leq C n, \quad n \geq 1
$$

where $C>0$ is some positive constant.

With these data, we define a scale of Banach spaces $B_{\rho}$ for $\rho>0$ by

$$
B_{\rho}=\left\{\left(v_{n}\right)_{n \geq 1} \in \prod_{n \geq 1} \mathcal{E}_{n} \mid\|v\|_{\rho}=\sum_{n \geq 1} \rho^{n}\left\|v_{n}\right\|_{n}<+\infty\right\} .
$$


We next define $F(t, v)=\left(\mathcal{S}_{n}(-t) \mathcal{C}_{n, n+1} \mathcal{S}_{n+1}(t) v_{n+1}\right)_{n \geq 1}$ for $t \in \mathbf{R}$. Observe that

$$
\begin{aligned}
\|F(t, v)\|_{\rho_{0}} \leq C \sum_{n \geq 1} n \rho_{0}^{n}\left\|v_{n}\right\|_{n} & \leq C \sum_{n \geq 1} \frac{\rho_{1}^{n}-\rho_{0}^{n}}{\rho_{1}-\rho_{0}}\left\|v_{n}\right\|_{n} \\
& \leq \frac{C}{\rho_{1}-\rho_{0}} \sum_{n \geq 1} \rho_{1}^{n}\left\|v_{n}\right\|_{n}=\frac{C\|v\|_{\rho_{1}}}{\rho_{1}-\rho_{0}} .
\end{aligned}
$$

In terms of $v_{n}(t)=\mathcal{S}_{n}(-t) u_{n}(t)$, the hierarchy (II.22) is recast as

$$
\dot{v}_{n}(t)=\mathcal{S}_{n}(-t) \mathcal{C}_{n, n+1} \mathcal{S}_{n+1}(t) v_{n+1}(t), \quad v_{n}(0)=u_{n}^{i n}, \quad n \geq 1,
$$

in other words,

$$
\dot{v}(t)=F(t, v(t)), \quad v(0)=\left(u_{n}^{i n}\right)_{n \geq 1} .
$$

Hence we arrive at the following uniqueness result:

Proposition II.3. Let $\left(u_{n}(t)\right)_{n \geq 1}$ be a solution to (II.22) such that, for each $T>0$, there exists $r(T)>0$ satisfying

$$
u_{n}(0)=0, \text { and } \sup _{t \in[0, T]}\left\|u_{n}(t)\right\|_{n} \leq r(T)^{n}, n \geq 1 .
$$

Then

$$
u_{n}(t)=0 \text { for each } t \in[0, T] \text { and each } n \geq 1 \text {. }
$$

\section{II.3. A digression on trace-class operators}

In order to apply the discussion above to the BBGKY hierarchy (II.13), we need to recall a few basic facts on trace-class operators.

Let $\mathfrak{H}$ be a separable Hilbert space; we denote by $\mathcal{L}(\mathfrak{H})$ the algebra of bounded operators on $\mathfrak{H}$ and by $\mathcal{K}(\mathfrak{H})$ the set of compact operators on $\mathfrak{H}$. The norm of an element $T$ of $\mathcal{L}(\mathfrak{H})$ is denoted by

$$
\|T\|=\sup _{\substack{\xi \in \mathfrak{s} \\|\xi|=1}}|T \xi|
$$

We recall that $\mathcal{K}(\mathfrak{H})$ is a closed two-sided ideal of $\mathcal{L}(\mathfrak{H})$ - more precisely $\mathcal{K}(\mathfrak{H})$ is the norm closure in $\mathcal{L}(\mathfrak{H})$ of the set of finite rank operators on $\mathfrak{H}$.

A bounded operator $T$ on $\mathfrak{H}$ is of trace-class if

$$
\sup \left\{\sum_{n \geq 0}\left|\left(T e_{n} \mid f_{n}\right)\right| \mid\left(e_{n}\right)_{n \geq 0} \&\left(f_{n}\right)_{n \geq 1} \text { orthonormal basis of } \mathfrak{H}\right\}<+\infty
$$

The sup in the definition above is denoted by $\|T\|_{1}$ and is called "the trace norm of $T^{\prime \prime}$. The set of trace-class operators on $\mathfrak{H}$ is a two-sided ideal in $\mathcal{L}(\mathfrak{H})$ denoted by $\mathcal{L}^{1}(\mathfrak{H})$. Equipped with the trace-norm, $\mathcal{L}^{1}(\mathfrak{H})$ is a Banach space, the completion of finite-rank operators on $\mathfrak{H}$ with respect to the trace-norm - in particular, both 
$\mathcal{L}^{1}(\mathfrak{H})$ and $\mathcal{K}(\mathfrak{H})$ are separable, unlike $\mathcal{L}(\mathfrak{H})$. In particular, every trace-class operator on $\mathfrak{H}$ is compact:

$$
\mathcal{L}^{1}(\mathfrak{H}) \subset \mathcal{K}(\mathfrak{H})
$$

For any $T \in \mathcal{L}^{1}(\mathfrak{H})$ and any $A \in \mathcal{L}(\mathfrak{H})$, both $A T$ and $T A$ belong to $\mathcal{L}^{1}(\mathfrak{H})$ and one has

$$
\|A T\|_{1}=\|T A\|_{1} \leq\|A\|\|T\|_{1} .
$$

If $T$ is a trace-class operator on $\mathfrak{H}$, its trace is

$$
\operatorname{trace}(T)=\sum_{n \geq 0}\left(T e_{n} \mid e_{n}\right) \text { where }\left(e_{n}\right)_{n \geq 1} \text { is any orthonormal basis of } \mathfrak{H}
$$

(the sum above being indeed independent of the choice of the orthonormal basis $\left.\left(e_{n}\right)_{n \geq 1}\right)$. Obviously, for each $T \in \mathcal{L}^{1}(\mathfrak{H})$,

$$
|\operatorname{trace}(T)| \leq\|T\|_{1} .
$$

In addition, if $A \in \mathcal{L}(\mathfrak{H})$ and $T \in \mathcal{L}^{1}(\mathfrak{H})$, then $[A, T] \in \mathcal{L}^{1}(\mathfrak{H})$ and

$$
\operatorname{trace}([A, T])=0 \text {. }
$$

The dual of $\mathcal{K}(\mathfrak{H})$ (viewed as a closed subspace of $\mathcal{L}(\mathfrak{H})$ for the operator norm) is canonically identified to $\mathcal{L}^{1}(\mathfrak{H})$, by viewing any trace-class operator $T$ as the continuous linear functional on $\mathcal{K}(\mathfrak{H})$ defined by

$$
K \mapsto \operatorname{trace}(T K)
$$

Likewise, the dual of the Banach space $\left(\mathcal{L}^{1}(\mathfrak{H}),\|\cdot\|_{1}\right)$ is canonically identified with $\mathcal{L}(\mathfrak{H})$ by viewing any bounded operator $A$ on $\mathfrak{H}$ as the continuous linear functional on $\mathcal{L}^{1}(\mathfrak{H})$ defined by

$$
T \mapsto \operatorname{trace}(A T) .
$$

This situation is identical to that of $c_{0}(\mathbf{N})$ (the set of vanishing sequences), whose dual is $\ell^{1}(\mathbf{N})$ (the class of summable sequences), which itself has $\ell^{\infty}(\mathbf{N})$ (the class of bounded sequences) as dual space. In particular the weak-* topology on $\mathcal{L}^{1}(\mathfrak{H})$ is that induced by the family of semi-norms

$$
p_{K}(T)=|\operatorname{trace}(T K)|
$$

where $K$ runs through $\mathcal{K}(\mathfrak{H})$.

Another important class of operators on $\mathfrak{H}$ is that of Hilbert-Schmidt operators, denoted by $\mathcal{L}^{2}(\mathfrak{H})$; a bounded operator $T$ on $\mathfrak{H}$ is in $\mathcal{L}^{2}$ if and only if $T^{*} T \in \mathcal{L}^{1}(\mathfrak{H})$. The class $\mathcal{L}^{2}(\mathfrak{H})$ is a two-sided ideal of $\mathcal{L}(\mathfrak{H})$ and a Banach space for the norm $\|T\|_{2}=\left\|T^{*} T\right\|_{1}^{1 / 2}$. Since $\mathcal{L}^{1}(\mathfrak{H})$ is an ideal in $\mathcal{L}(\mathfrak{H}), \mathcal{L}^{1}(\mathfrak{H}) \subset \mathcal{L}^{2}(\mathfrak{H})$.

Finally, we consider the special case where $\mathfrak{H}=L^{2}\left(\mathbf{R}^{D}\right)$. There is an extremely simple characterization of $\mathcal{L}^{2}\left(\mathbf{R}^{D}\right)$ : an operator $T \in \mathcal{L}^{2}\left(\mathbf{R}^{D}\right)$ if and only if it is an integral operator of the form

$$
(T f)(x)=\int k_{T}(x, y) f(y) d y \text { with } k_{T} \in L^{2}\left(\mathbf{R}^{D} \times \mathbf{R}^{D}\right) .
$$


If $T$ is a trace-class operator on $\mathfrak{H}$, it is a Hilbert-Schmidt operator and as such is defined as above by an integral kernel $k_{T} \in L^{2}\left(\mathbf{R}^{D} \times \mathbf{R}^{D}\right)$. But this integral kernel has additional regularity properties: in particular, if $T \in \mathcal{L}^{1}\left(\mathbf{R}^{D}\right)$, the function

$$
(z, h) \mapsto k_{T}(z+h, z) \text { belongs to } C_{b}\left(\mathbf{R}_{h}^{D} ; L^{1}\left(\mathbf{R}_{z}^{D}\right)\right) .
$$

Consider further the case where $\mathfrak{H}=L^{2}\left(\mathbf{R}^{m} \times \mathbf{R}^{n}\right)$, and let $T \in \mathcal{L}^{1}(\mathfrak{H})$ with integral kernel $k_{T} \equiv k_{T}\left(x_{1}, x_{2}, y_{1}, y_{2}\right)$, with $x_{1}, y_{1} \in L^{2}\left(\mathbf{R}^{m}\right)$ while $x_{2}, y_{2} \in L^{2}\left(\mathbf{R}^{n}\right)$. For a.e. $x_{2}, y_{2}$, let $T_{x_{2}, y_{2}}$ be the operator on $\mathcal{L}^{1}\left(L^{2}\left(\mathbf{R}^{m}\right)\right)$ with integral kernel $\left(x_{1}, y_{1}\right) \mapsto$ $k_{T}\left(x_{1}, x_{2}, y_{1}, y_{2}\right)$. Then

(1) the map

$$
(z, h) \mapsto T_{z+h, z} \text { belongs to } C_{b}\left(\mathbf{R}_{h}^{n}, L^{1}\left(\mathbf{R}_{z}^{m} ; \mathcal{L}^{1}\left(L^{2}\left(\mathbf{R}^{D}\right)\right)\right)\right) ;
$$

(2) in particular, for a.e. $z \in \mathbf{R}^{n}$, one has $T_{z, z} \in \mathcal{L}^{1}\left(L^{2}\left(\mathbf{R}^{m}\right)\right)$ and

$$
\left\|T_{z, z}\right\|_{1} \leq\|T\|_{1} .
$$

For these last results, see [5], Lemmas 2.1 and 2.3.

\section{II.4. Application to the Hartree approximation}

The precise mathematical statement of the mean-field limit for the $N$-body Schrödinger equation leading to Hartree's equation is summarized in the following

Theorem II.4. Let $\psi^{0} \in H^{1}\left(\mathbf{R}^{D}\right)$ with $\left\|\psi^{0}\right\|_{L^{2}}=1$, and let $\psi$ be the solution to Hartree's equation

$$
\begin{aligned}
i \partial_{t} \psi(t, x)+\frac{1}{2} \Delta_{x} \psi(t, x) & =\psi(t, x) \int V(x-y)|\psi(t, y)|^{2} d y \\
\psi(0, x) & =\psi^{0}(x) .
\end{aligned}
$$

For each $N \geq 1$, let $\Psi_{N}$ be the solution to the $N$-body Schrödinger equation

$$
\begin{aligned}
i \partial_{t} \Psi_{N}\left(t, X_{N}\right)+\frac{1}{2} \sum_{k=1}^{N} \Delta_{x_{k}} \Psi_{N}\left(t, X_{N}\right) & =\frac{1}{N_{1 \leq k<l \leq N}} \sum_{1 \leq N} V\left(x_{k}-x_{l}\right) \Psi_{N}\left(t, X_{N}\right), \\
\Psi_{N}(0, x) & =\prod_{k=1}^{N} \psi^{0}\left(x_{k}\right) .
\end{aligned}
$$

Then, for each $n \geq 1$ and each $t>0$, the reduced density matrix

$$
\int \Psi_{N}\left(t, X_{n}, Z_{n}^{N}\right) \overline{\Psi_{N}\left(t, Y_{n}, Z_{n}^{N}\right)} d Z_{n}^{N} \rightarrow \prod_{k=1}^{n} \psi\left(t, x_{k}\right) \overline{\psi\left(t, y_{k}\right)}
$$

in the weak-* topology of $\mathcal{L}^{1}\left(L^{2}\left(\left(\mathbf{R}^{D}\right)^{n}\right)\right)$ - identifying trace-class operators with their integral kernels - as $N \rightarrow+\infty$.

H. Spohn sketched a first proof of this result in his very interesting review article [30]; we refer to [5] for a more complete proof. 
Proof. The proof is divided in several steps.

Step 1. Since $\Psi_{N}^{0} \in L^{2}\left(\left(\mathbf{R}^{D}\right)^{N}\right)$ with $\left\|\Psi_{N}^{0}\right\|_{L^{2}}=1$, the solution $\Psi_{N} \equiv \Psi_{N}\left(t, X_{N}\right)$ to the $N$-body Schrödinger equation satisfies $\left\|\Psi_{N}(t, \cdot)\right\|_{L^{2}}=1$ for all $t \in \mathbf{R}$. Hence $D_{N}(t) \geq 0$ in $\mathcal{L}^{1}\left(L^{2}\left(\left(\mathbf{R}^{D}\right)^{N}\right)\right)$ and one has

$$
\operatorname{trace}\left(D_{N}(t)\right)=\left\|D_{N}(t)\right\|_{1}=1 .
$$

Hence, for all $n \geq 1, D_{N: n}(t) \geq 0$, belongs to $\mathcal{L}^{1}\left(L^{2}\left(\left(\mathbf{R}^{D}\right)^{n}\right)\right)$, and one has

$$
\operatorname{trace}\left(D_{N: n}(t)\right)=\left\|D_{N: n}(t)\right\|_{1}=1
$$

Therefore, the sequence indexed by $N \geq 1$ of marginal distributions $\left(D_{N: n}\right)_{n \geq 1}$ is relatively compact in $\prod_{n>1} L^{\infty}\left(\mathbf{R} ; \mathcal{L}^{1}\left(L^{2}\left(\left(\mathbf{R}^{D}\right)^{n}\right)\right)\right)$ equip- ped with the product of weak-* topologies on each factor. Let $\left(D_{n}\right)_{n \geq 1}$ be one of its limit points: there exists a subsequence $N_{k} \rightarrow+\infty$ such that, for each $n \geq 1$

$$
D_{N_{k}: n} \rightarrow D_{n} \text { weakly-* in } L^{\infty}\left(\mathbf{R} ; \mathcal{L}^{1}\left(L^{2}\left(\left(\mathbf{R}^{D}\right)^{n}\right)\right)\right) \text { as } N_{k} \rightarrow+\infty .
$$

Step 2. Let $\left(D_{n}\right)_{n \geq 1}$ be a limit point of $\left(D_{N: n}\right)_{n \geq 1}$; we are going to prove that $\left(D_{n}\right)_{n \geq 1}$ is a solution to the infinite hierarchy (II.15). First,

$$
\begin{aligned}
& i \partial_{t} D_{N: n}(\left.t, X_{n}, Y_{n}\right)+\frac{1}{2} \sum_{k=1}^{n}\left(\Delta_{x_{k}}-\Delta_{y_{k}}\right) D_{N: n}\left(t, X_{n}, Y_{n}\right) \\
& \rightarrow i \partial_{t} D_{n}\left(t, X_{n}, Y_{n}\right)+\frac{1}{2} \sum_{k=1}^{n}\left(\Delta_{x_{k}}-\Delta_{y_{k}}\right) D_{n}\left(t, X_{n}, Y_{n}\right)
\end{aligned}
$$

in the sense of distributions on $\mathbf{R} \times\left(\mathbf{R}^{D}\right)^{n} \times\left(\mathbf{R}^{D}\right)^{n}$. Next, we estimate the so-called "re-collision term"

$$
\begin{array}{r}
\left\|\frac{1}{N} \sum_{1 \leq k<l \leq n}\left(V\left(x_{k}-x_{l}\right)-V\left(y_{k}-y_{l}\right)\right) D_{N: n}\left(t, X_{n}, Y_{n}\right)\right\|_{L_{t}^{\infty}\left(L_{X_{n}, Y_{n}}^{2}\right)} \\
\leq \frac{n(n-1)}{N}\|V\|_{L^{\infty}}\left\|D_{N: n}\right\|_{L_{t}^{\infty}\left(L_{X_{n}, Y_{n}}^{2}\right)}
\end{array}
$$

so that

$$
\frac{1}{N} \sum_{1 \leq k<l \leq n}\left(V\left(x_{k}-x_{l}\right)-V\left(y_{k}-y_{l}\right)\right) D_{N: n}\left(t, X_{n}, Y_{n}\right) \rightarrow 0
$$

in $L^{\infty}\left(\mathbf{R} ; L^{2}\left(\left(\mathbf{R}^{D}\right)^{n} \times\left(\mathbf{R}^{D}\right)^{n}\right)\right.$. Finally, for each $k=1, \ldots, n$, the interaction term satisfies

$$
\begin{aligned}
\int V\left(x_{k}-z\right) D_{N_{k}: n+1} & \left(t, X_{n}, z, Y_{n}, z\right) d z \\
& \rightarrow \int V\left(x_{k}-z\right) D_{n+1}\left(t, X_{n}, z, Y_{n}, z\right) d z
\end{aligned}
$$


and similarly

$$
\begin{aligned}
\int D_{N_{k}: n+1}\left(t, X_{n}, z, Y_{n}, z\right) & V\left(y_{k}-z\right) d z \\
& \rightarrow \int D_{n+1}\left(t, X_{n}, z, Y_{n}, z\right) V\left(y_{k}-z\right) d z
\end{aligned}
$$

weakly-* in $L^{\infty}\left(\mathbf{R} ; \mathcal{L}^{1}\left(L^{2}\left(\left(\mathbf{R}^{D}\right)^{n}\right)\right)\right)$ as $N_{k} \rightarrow+\infty$. Notice that the map

$$
\rho_{n+1} \mapsto \int\left(V\left(x_{k}-z\right)-V\left(y_{k}-z\right)\right) \rho_{n+1}\left(X_{n}, z, Y_{n}, z\right) d z
$$

is continuous from $\mathcal{L}^{1}\left(L^{2}\left(\mathbf{R}^{D}\right)^{n+1}\right)$ to $\mathcal{L}^{1}\left(L^{2}\left(\mathbf{R}^{D}\right)^{n}\right)$.

This continuity property would be enough if we knew that $D_{N_{k}: n+1}(t)$ converges to $D_{N_{k}: n}(t)$ weakly in $\mathcal{L}^{1}\left(L^{2}\left(\left(\mathbf{R}^{D}\right)^{n}\right)\right.$, but we only know that this sequence converges weakly-* in in $\mathcal{L}^{1}\left(L^{2}\left(\left(\mathbf{R}^{D}\right)^{n}\right)\right.$. The limits above hold indeed, but their proof requires additional work. After integrating the $n$-th equation in the BBGKY hierarchy against some test function, the convergence property to be proved ultimately rests on the following lemma (Lemma 2.3 in [5], to which we refer for a proof).

Lemma II.5. Consider a sequence $T_{N} \in \mathcal{L}^{1}\left(L^{2}\left(\mathbf{R}^{D}\right)\right)$ that converges to 0 weakly-* and whose (sequence of) integral kernels $k_{T_{N}}$ satisfies

$$
\int\left|k_{T_{N}}(z, z+h)-k_{T_{N}}(z, z)\right| d z \rightarrow 0 \text { as }|h| \rightarrow 0 \text { uniformly in } N \text {. }
$$

Then, for any $\chi \in C_{c}\left(\mathbf{R}^{D}\right)$

$$
\int \chi(z) k_{T_{N}}(z, z) d z \rightarrow 0 \text { as } N \rightarrow+\infty .
$$

Hence, passing to the limit as $N_{k} \rightarrow+\infty$ in each equation of the BBGKY hierarchy (II.13) shows that $\left(D_{n}\right)_{n \geq 1}$ is a solution to the infinite hierarchy (II.15). The uniform convergence in $N$ required in the Lemma above comes from the $H^{1}$ bound implied by the conservation of energy and the symmetry (II.12) preserved under the evolution of the $N$-body linear Schrödinger equation.

Finally, observe that, for each $\zeta \in C_{c}^{\infty}\left(\mathbf{R}^{n} \times \mathbf{R}^{n}\right)$,

$$
\partial_{t} \iint D_{N: n}\left(t, X_{n}, Y_{n}\right) \zeta\left(X_{n}, Y_{n}\right) d X_{n} d Y_{n} \text { is bounded in } L^{\infty}\left(\mathbf{R}_{+}\right)
$$

(this follows from integrating by parts to bring the Laplacian operators in the $n$ th equation of (II.13) to bear on $\zeta$, and from the convergence properties of the re-collision and interaction terms above). This last bound establishes the initial condition (II.16).

Step 3. Suppose that $\left(D_{n}\right)_{n \geq 1}$ is a limit point of the sequence $\left(D_{N: n}\right)_{n \geq 1}$ as $N \rightarrow+\infty$ for the product of weak-* topologies in $\prod_{n \geq 1} L^{\infty}\left(\mathbf{R} ; \mathcal{L}^{1}\left(L^{2}\left(\left(\mathbf{R}^{D}\right)^{n}\right)\right)\right)$, then $\left(D_{n}\right)_{n \geq 1}$ is a solution to the infinite hierarchy (II.15) with initial data (II.16). 
As we observed earlier (see Proposition II.1), $\left(F_{n}\right)_{n \geq 1}$ defined by

$$
F_{n}\left(t, X_{n}, Y_{n}\right)=\prod_{1 \leq k \leq n} \psi\left(t, x_{k}\right) \overline{\psi\left(t, y_{k}\right)}
$$

where $\psi$ is the solution to Hartree's equation (II.9) is also a solution to (II.15)(II.16) .

Since the infinite hierarchy (II.15) is linear, $\left(Q_{n}\right)_{n \geq 1}$ defined for each $n \geq 1$ by $Q_{n}=D_{n}-F_{n}$ is a solution to (II.15) with initial data

$$
\left.Q_{n}\right|_{t=0} \equiv 0, \quad n \geq 1
$$

Further, one has

$$
\left\|Q_{n}(t)\right\|_{1} \leq\left\|D_{n}(t)\right\|_{1}+\left\|F_{n}(t)\right\|_{1} \leq 2 ;
$$

indeed, $\left\|D_{N: n}(t)\right\|_{1}=1$ so that, by convexity and weak-* convergence, $\left\|D_{n}(t)\right\|_{1} \leq$ 1 for each $n \geq 1$ and each $t \in \mathbf{R}$; likewise, for each $n \geq 1$ and each $t \in \mathbf{R}$, $\left\|F_{n}(t)\right\|_{1}=\|\psi(t, \cdot)\|_{L^{2}}^{2 n}=1$ since the dynamics of Hartree's equation (II.9) entails $\|\psi(t, \cdot)\|_{L^{2}}=\left\|\psi^{0}\right\|_{L^{2}}=1$.

Then we apply Proposition II.3 to the infinite BBGKY hierarchy (II.15) with

$$
\mathcal{A}_{n} D_{n}=-i \frac{1}{2} \sum_{k=1}^{n}\left(\Delta_{x_{k}}-\Delta_{y_{k}}\right) D_{n}
$$

and

$$
\begin{aligned}
& \left(\mathcal{C}_{n, n+1} D_{n+1}\right)\left(X_{n}, Y_{n}\right) \\
& \quad=\sum_{k=1}^{n} \int\left(V\left(x_{k}-z\right)-V\left(y_{k}-z\right)\right) D_{n+1}\left(X_{n}, z, Y_{n}, z\right) d z,
\end{aligned}
$$

on $\mathcal{E}_{n}=\mathcal{L}^{1}\left(L^{2}\left(\left(\mathbf{R}^{D}\right)^{n}\right)\right)$, for each $n \geq 1$. As noticed in the previous step, the inequality (II.26) implies that

$$
\left\|\mathcal{C}_{n, n+1}\right\|_{\mathcal{L}\left(\mathcal{E}_{n+1}, \mathcal{E}_{n}\right)} \leq n, \quad n \geq 1
$$

On the other hand, $\mathcal{A}_{n}$ generates on $\mathcal{E}_{n}$ the group of isometries defined by

$$
\mathcal{S}_{n}(t) D_{n}=e^{-t \mathcal{A}_{n}} D_{n}=e^{-i t \frac{1}{2}\left(\Delta_{1}+\ldots+\Delta_{n}\right)} D_{n} e^{i t \frac{1}{2}\left(\Delta_{1}+\ldots+\Delta_{n}\right)} .
$$

Applying Proposition II.3 shows that $Q_{n}(t) \equiv 0$ for all $n \geq 1$ and all $t \in \mathbf{R}$.

Hence the sequence $\left(D_{N: n}\right)_{n \geq 1}$ converges in $\prod_{n \geq 1} L^{\infty}\left(\mathbf{R} ; \mathcal{L}^{1}\left(L^{2}\left(\left(\mathbf{R}^{D}\right)^{n}\right)\right)\right)$ with the product of weak-* topologies to $\left(F_{n}\right)_{n \geq 1}$ as $N \rightarrow+\infty$.

\section{Part III. From the quantum $N$-body problem to the TDHF equations}

\section{III.1. Background on Fermi particles}

In our derivation of Hartree's equation, we postulated that the initial data for the $N$-body, linear Schrödinger equation (II.3) is factorized, i.e. of the form (II.7). This 
means that the $N$ particles considered are all in the same quantum state defined by the 1-body wave function $\psi^{0}$.

If the particles considered are fermions (such as electrons, positrons, protons, and more generally particles with spin $1 / 2$ ), such initial data are ruled out by Pauli's exclusion principle - which says that two such particles cannot be in the same quantum state. (Actually, this counting takes into account the spin variable, unlike in our present discussion: for instance, one could find two electrons in what we consider here as one quantum state, one with spin up, one with spin down).

In any case, for a system of $N$ fermions, the wave function should have the following symmetry property:

$$
\Psi_{N}\left(X_{N}\right)=\operatorname{sign}(\sigma) \Psi_{N}\left(x_{\sigma(1)}, \ldots, x_{\sigma(N)}\right), \quad \sigma \in \mathfrak{S}_{N} .
$$

On the contrary, for a system of $N$ bosons (particles such as photons, $\alpha$ particles or more generally particles with integer spin), the wave function should have the symmetry

$$
\Psi_{N}\left(X_{N}\right)=\Psi_{N}\left(x_{\sigma(1)}, \ldots, x_{\sigma(N)}\right), \quad \sigma \in \mathfrak{S}_{N} .
$$

The case considered in the previous section (namely $N$ particles in the same quantum state defined by the wave function $\psi^{0}$ ) corresponds to the case of Bose condensation.

An important example of $N$-particle wave function with symmetry (III.1) is the case of Slater determinants. A Slater determinant is a $N$-body wave function of the form

$$
\Psi_{N}\left(X_{N}\right)=\frac{1}{\sqrt{N !}} \operatorname{det}\left(\psi_{k}\left(x_{l}\right)\right)_{1 \leq k, l \leq N}
$$

where $\left(\psi_{k}\right)_{1 \leq k \leq N}$ is an orthonormal system in $L^{2}\left(\mathbf{R}^{D}\right)$ :

$$
\int \psi_{k}(x) \overline{\psi_{l}(x)} d x=\delta_{k l}, \quad 1 \leq k, l \leq N
$$

Slater determinants are the wave functions of non-interacting systems of fermions. We leave it to the reader to verify the following formulas:

\section{REDUCED DENSITY MATRICES FOR SlATER DETERMINANTS}

If $D_{N}\left(X_{N}, Y_{N}\right)=\Psi_{N}\left(X_{N}\right) \overline{\Psi_{N}\left(Y_{N}\right)}$ with $\Psi_{N}$ a Slater determinant (III.3), then its reduced density matrices are

$$
D_{N: 1}(x, y)=\frac{1}{N} \sum_{k=1}^{N} \psi_{k}(x) \psi_{k}(y)^{*}
$$

while the next reduced density matrices are all expressed in terms of this first one:

$$
D_{N: j}(x, y)=\frac{(N-k) ! N^{k}}{N !} \operatorname{det}\left(D_{N: 1}\left(x_{k}, y_{l}\right)\right)_{1 \leq k, l \leq N}
$$

We leave it to the reader as an exercise to verify these formulas - in fact, there is a quick argument that explains why the factor $\frac{(N-k) ! N^{k}}{N !}$ should be greater than or equal to 1 (hint: compute the trace of each side of the equality above). 
Notice that

$$
D_{N: 1} \geq 0, \quad \operatorname{trace}\left(D_{N: 1}\right)=1, \quad\left\|D_{N: 1}\right\|_{\mathcal{L}(\mathfrak{H})}=\frac{1}{N} \rightarrow 0
$$

as $N \rightarrow+\infty$.

We conclude this discussion with a remark concerning the statistics of the $N$ particles (bosons or fermions). For each $\sigma \in \mathfrak{S}_{N}$, define $U_{\sigma}$ by the formula

$$
\left(U_{\sigma} f\right)\left(X_{N}\right)=f\left(x_{\sigma^{-1}(1)}, \ldots, x_{\sigma^{-1}(N)}\right) .
$$

With this notation, the symmetry property that characterizes Fermi particles (III.1) reads

$$
\left(U_{\sigma} \Psi_{N}\right)\left(X_{N}\right)=\operatorname{sign}(\sigma) \Psi_{N}\left(X_{N}\right), \quad \sigma \in \mathfrak{S}_{N} .
$$

On the other hand, if the $N$ particles considered are bosons, their wave function has the symmetry

$$
\left(U_{\sigma} \Psi_{N}\right)\left(X_{N}\right)=\Psi_{N}\left(X_{N}\right), \quad \sigma \in \mathfrak{S}_{N}
$$

In both cases, the associated density matrix has the symmetry

$$
\begin{array}{r}
D_{N}\left(x_{\sigma^{-1}(1)}, \ldots, x_{\sigma^{-1}(N)}, y_{\sigma^{-1}(1)}, \ldots, y_{\sigma^{-1}(N)}\right) \\
=D_{N}\left(X_{N}, Y_{N}\right), \quad \sigma \in \mathfrak{S}_{N}
\end{array}
$$

that characterizes systems of $N$ undistinguishable particles. In the language of operators, the density operator of a system of $N$ undistinguishable particles satisfies

$$
D_{N}=U_{\sigma} D_{N} U_{\sigma}^{*}
$$

moreover

$$
\begin{array}{ll}
U_{\sigma} D_{N}=D_{N} U_{\sigma}=D_{N}, & \text { in the case of bosons, } \\
U_{\sigma} D_{N}=D_{N} U_{\sigma}=\operatorname{sign}(\sigma) D_{N}, & \text { in the case of fermions. }
\end{array}
$$

\section{III.2. The TDHF equation in operator form}

Suppose we are given $N$ orbitals $\psi_{1}(t, x), \ldots, \psi_{N}(t, x)$ that satisfy the TDHF system

$$
\begin{aligned}
& i \partial_{t} \psi_{k}(t, x)=-\frac{1}{2} \Delta \psi_{k}(t, x) \\
+ & \frac{1}{N} \sum_{l=1}^{N} \psi_{k}(t, x) \int V(|x-y|)\left|\psi_{l}(t, y)\right|^{2} d y \\
- & \frac{1}{N} \sum_{l=1}^{N} \psi_{l}(t, x) \int V(|x-y|) \psi_{k}(t, y) \overline{\psi_{l}(t, y)} d y, \quad 1 \leq k \leq N,
\end{aligned}
$$

and is, for each $t \geq 0$, an orthonormal system in $L^{2}\left(\mathbf{R}^{3}\right)$ :

$$
\int \psi_{k}(t, x) \overline{\psi_{l}(t, x)} d x=\delta_{k l}, \quad 1 \leq k, l \leq N .
$$


We recall that $V$ is assumed to be real-valued; a straightforward computation shows that if the initial orbitals $\psi_{1}(0, \cdot), \ldots, \psi_{N}(0, \cdot)$ satisfy (III.15), then $\psi_{1}(t, \cdot), \ldots, \psi_{N}(t, \cdot)$ satisfy (III.15) for each $t>0$.

With the system of $N$ orbitals above, we define the 1-particle density matrix

$$
F(t, x, y)=\frac{1}{N} \sum_{k=1}^{N} \psi_{k}(t, x) \overline{\psi_{k}(t, y)}
$$

— in the language of operators

$$
F(t)=\frac{1}{N} \sum_{k=1}^{N}\left|\psi_{k}(t, \cdot)\right\rangle\left\langle\psi_{k}(t, \cdot)\right| .
$$

If the $N$ orbitals $\psi_{1}(t, x), \ldots, \psi_{N}(t, x)$ satisfy (III.14), the 1-particle density matrix $F$ satisfies

$$
\begin{aligned}
i \partial_{t} F(t, x, y) & +\frac{1}{2}\left(\Delta_{x}-\Delta_{y}\right) F(t, x, y) \\
& =F(t, x, y) \int(V(x-z)-V(y-z)) F(t, z, z) d z \\
& -\int(V(x-z)-V(y-z)) F(t, x, z) F(t, z, y) d z
\end{aligned}
$$

Let us recast this equation in the language of operators: denote by $U_{1,2}$ the unitary operator on $\mathfrak{H}^{\otimes 2}$ associated to the transposition that exchanges 1 and 2 by the definition (III.8). In other words, $U_{1,2} \xi \otimes \eta=\eta \otimes \xi$; equivalently, $U_{12} \phi\left(x_{1}, x_{2}\right)=\phi\left(x_{2}, x_{1}\right)$. To the potential $V$, we associate the operator acting on $\mathfrak{H}^{\otimes 2}$ by multiplication:

$$
V: \phi\left(x_{1}, x_{2}\right) \mapsto V\left(x_{1}-x_{2}\right) \phi\left(x_{1}, x_{2}\right) .
$$

With these notations, the equation (III.18) is recast as

$$
i \partial_{t} F+\frac{1}{2}[\Delta, F]=\left[V, F^{\otimes 2}\left(I-U_{12}\right)\right]_{: 1} .
$$

The TDHF equation has been studied by various authors; the following result showing that the Cauchy problem for (III.19) is well-posed is due to Bove-DaPratoFano [6].

Theorem III.1. Let $V$ be an even, real-valued function in $L^{\infty}\left(\mathbf{R}^{D} \times \mathbf{R}^{D}\right)$. Let $F^{0} \in \mathcal{L}^{1}(\mathfrak{H})$ be such that

$$
F^{0} \geq 0, \quad \operatorname{trace}\left(F^{0}\right)=1 .
$$

Then, there exists a unique mild solution $F \equiv F(t)$ to (III.19) such that $F(0)=F^{0}$. Further, for all $t \geq 0$, there exists a unitary operator $u(t)$ on $\mathfrak{H}$ such that

$$
F(t)=u(t) F(0) u(t)^{*}, \quad t \geq 0 .
$$

In particular, one has, for each $t \geq 0$,

$$
F(t) \geq 0, \quad \operatorname{trace}(F(t))=1, \quad\|F(t)\|=\left\|F^{0}\right\| .
$$


Notice that $F(t)$ depends on $N$, however we opted against the notation $F_{N}(t)$ to emphasize the fact that $F(t)$ is a 1-particle object.

Electrons being the first example of Fermi particles, thus it is natural to consider the TDHF equation with Coulomb (instead of bounded) 2-body interaction. The analogue of Theorem III.1 in the Coulomb case has been proved by Glassey-Chadam [12] in orbital form, and later generalized by Bove-DaPrato-Fano [7] in operator form (see also [10]). Comparing both proofs shows the rationale for working on the TDHF equation in operator (rather than orbital) form. On the other hand, numerical treatments of the Hartree-Fock equations is usually done in terms of the operator, rather than on the orbital formulation.

\section{III.3. Convergence of the $N$-body problem to the TDHF equation}

Before stating our main result on this problem, we introduce the following terminology.

Definition III.2. A sequence $D_{N} \in \mathcal{L}^{1}\left(\mathfrak{H}^{\otimes M}\right)$ is said to have Slater closure if and only if, for each $j \geq 1$,

$$
\left\|D_{N: j}-D_{N: 1}^{\otimes j} \Sigma_{j}\right\|_{1} \rightarrow 0 \quad \text { as } N \rightarrow+\infty,
$$

where $\Sigma_{j}$ designates the operator

$$
\Sigma_{j}=\sum_{\sigma \in \mathfrak{S}_{j}} \operatorname{sign}(\sigma) U_{\sigma}
$$

The elementary formulas (III.5) and (III.6) show that pure Slater states have indeed Slater closure - which justifies this terminology.

The TDHF approximation for bounded 2-body interaction is summarized in the following Theorem, taken from [3]:

Theorem III.3. Let $V$ be an even, real-valued function in $L^{\infty}\left(\mathbf{R}^{D} \times \mathbf{R}^{D}\right)$. For each $N \geq 1$, let $\psi_{1}^{0}, \ldots, \psi_{N}^{0}$ be an orthonormal system in $\mathfrak{H}$. Let $\Psi_{N}^{0}$ be the Slater determinant built on the $\psi_{j}^{0}$ s, let $\Psi_{N}\left(t, X_{N}\right)$ be the solution to the $N$-body Schrödinger equation (II.3) with initial data $\Psi_{N}^{0}$, and let $D_{N}(t)$ be the pure state defined by

$$
D_{N}(t)=\left|\Psi_{N}(t, \cdot)\right\rangle\left\langle\Psi_{N}(t, \cdot)\right|
$$

Finally, let $F(t)$ be the solution to the TDHF equation (III.19) with initial data

$$
F^{0}=\frac{1}{N} \sum_{k=1}^{N}\left|\psi_{k}(t, \cdot)\right\rangle\left\langle\psi_{k}(t, \cdot)\right|
$$

Then, as $N \rightarrow+\infty$,

$$
\left\|D_{N: j}(t)-F(t)^{\otimes j} \Sigma_{j}\right\|_{1} \rightarrow 0
$$

for each $j \geq 1$ and each $t \geq 0$. 
Observe that this result shows in particular that $D_{N}(t)$ has Slater closure for each $t>0$. In fact, the result in [3] shows that the same result holds verbatim if $D_{N}(t)$ is the solution of the $N$-body von Neumann equation (II.6) with initial data $D_{N}(0)$ having Slater closure, while $F(t)$ is the solution to the TDHF equation (III.19) with initial data $D_{N: 1}(0)$.

Hence, while the mean-field limits leading to the Vlasov or Hartree equations were based on the propagation of chaos - as defined in Appendix B of part I the convergence to the TDHF equation rests on the propagation of Slater closure, in the sense of the definition above.

\section{III.4. Sketch of the proof of the TDHF approximation}

The interested reader is referred to [3] for a complete proof; here we just sketch the main ideas in it.

Proof of Theorem III.3. Step 1. Observe that $D_{N}(0)$ has the symmetry (III.12); hence $D_{N}(t)$ also satisfies (III.12), since the 2-body interaction is an even function $V$. As explained in part II, this symmetry property implies that the sequence of reduced density operators $D_{N: j}(t)$ satisfies the BBGKY hierarchy (II.13) for $1 \leq j \leq N$.

However, unlike in the case of Hartree's approximation, the sequence $\left(F_{j}^{-}\right)_{j \geq 1}$ defined by the formula

$$
F_{j}^{-}(t)=F(t)^{\otimes j} \Sigma_{j}, \quad j \geq 1,
$$

is usually not a solution to the infinite BBGKY hierarchy (II.15). This property was crucial in the proof of Hartree's approximation; for the TDHF approximation however, a different type of argument is required.

Step 2: the TDHF hierarchy. Although $\left(F_{j}^{-}\right)_{j \geq 1}$ is not a solution to (II.15), it solves a different (hopefully slightly different) infinite hierarchy of equations. This infinite hierarchy, built "from the bottom up", starting from the TDHF equation (III.19) satisfied by $F(t)$ is then compared to (II.15).

Straightforward computations show that this infinite hierarchy, henceforth referred to as the TDHF hierarchy, is

$$
i \partial_{t} F_{j}^{-}+\frac{1}{2} \sum_{k=1}^{j}\left[\Delta_{k}, F_{j}^{-}\right]=\sum_{k=1}^{j}\left[V_{k, j+1}, F_{j+1}^{-}\right]_{: j}+\mathcal{R}_{j}(F), \quad j \geq 1,
$$

where $\Delta_{k}=I^{\otimes k-1} \otimes \Delta \otimes I^{\otimes j-k}$ and where the remainders $\mathcal{R}_{j}(F)$ are defined as follows:

$$
\begin{aligned}
& \mathcal{R}_{1}(F)=0 \\
& \mathcal{R}_{j}(F)=\sum_{k=1}^{j}\left[V_{k, j+1}, F^{\otimes j+1} \sum_{\substack{l=1 \\
k \neq l}}^{j} U_{l, j+1}\right]_{: j} \Sigma_{j}, \quad j>1 .
\end{aligned}
$$

At first sight, there seems to be no obvious reason for separating $\mathcal{R}_{j}(F)$ from $\sum_{k=1}^{j}\left[V_{k, j+1}, F_{j+1}^{-}\right]_{: j}$ in the interaction term, and for viewing the latter term as the leading order and the former term as a remainder. 
Step 3: consistency. Here we use the language of Numerical Analysis - specifically, of Lax's equivalence theorem: in order to prove that a numerical scheme converges, one proves that it is consistent and stable. Consistency means that the discretized equation "approximates" the exact equation, in the sense that any smooth solution of the exact equation nearly satisfies the discretized equation.

In the present case, consistency means that the infinite TDHF hierarchy approximates the BBGKY hierarchy. The key observation in this direction is the following

Proposition III.4. Let $V$ be a real-valued, even function in $L^{\infty}\left(\mathbf{R}^{D} \times \mathbf{R}^{D}\right)$ and let $F \in \mathcal{L}^{1}(\mathfrak{H})$. Then, for each $j \geq 1$,

$$
\left\|\mathcal{R}_{j}(F)\right\|_{1} \leq 2 j(j-1)\|V\|_{L^{\infty}}\|F\|\left\|F_{j}^{-}\right\|_{1} .
$$

Let us postpone momentarily the proof of this Proposition, and explain how it is used in the proof of Theorem III.3.

The Proposition above shows that

$$
\left\|\mathcal{R}_{j}(F(t))\right\|_{1} \leq 2\|V\|_{L^{\infty}} \frac{j(j-1)}{N}, \text { for all } j \geq 1,
$$

since a straightforward computation shows that

$$
\left\|F_{j}^{-}(t)\right\|_{1} \leq\|F(t)\|_{1}^{j}=1, \quad \text { for all } j \geq 1,
$$

while the last statement in Theorem III.1 shows that

$$
\|F(0)\|=\frac{1}{N} .
$$

Hence, for each fixed $j, \mathcal{R}_{j}(F(t)) \rightarrow 0$ as $N \rightarrow+\infty$, uniformly in $t \geq 0$. In this sense, the infinite TDHF hierarchy (III.21) is an approximation to the BBGKY hierarchy (II.13).

Step 4: stability. Define

$$
\mathcal{A}_{j} D_{j}=\sum_{k=1^{j}}\left[\frac{1}{2} \Delta_{k}, D_{j}\right]
$$

and

$$
\mathcal{C}_{j, j+1} D_{j+1}=\sum_{k=1}^{j}\left[V_{k, n+1}, D_{j+1}\right]_{: j} .
$$

We are interested in controlling the difference $E_{N, j}=D_{N: j}-F_{j}^{-}$, with $D_{N: j} \equiv 0$ whenever $j>N$. It follows from steps 1 and 2 that $E_{N, j}$ satisfies the hierarchy of equations

$$
\partial_{t} E_{N, j}=i \mathcal{A}_{j} E_{N, j}-i \mathcal{C}_{j, j+1} E_{N, j+1}-i \epsilon_{N, j}
$$

where

$$
\begin{aligned}
\epsilon_{N, j}(t) & =\frac{1}{N} \sum_{1 \leq k<l \leq j}\left[V_{k l}, D_{N: j}(t)\right] \\
& -\frac{j}{N} \sum_{k=1}^{j}\left[V_{k, n+1}, D_{N: j+1}(t)\right]_{: j}-\mathcal{R}_{j}(F(t)) .
\end{aligned}
$$


In the consistency error term $\epsilon_{N, j}$, the two first terms come from the difference between the BBGKY hierarchy (II.13) and the infinite BBGKY hierarchy (II.15); the last term comes from the difference between the infinite BBGKY hierarchy (II.15) and the infinite TDHF hierarchy (III.21).

Starting from Duhamel's formula

$$
\begin{aligned}
E_{N, j}(t)=\mathcal{S}_{j}(t) E_{N, j}(0) & -i \int_{0}^{t} \mathcal{S}_{j}\left(t-t_{1}\right) \epsilon_{N, j}\left(t_{1}\right) d t_{1} \\
& -i \int_{0}^{t} \mathcal{S}_{j}\left(t-t_{1}\right) \mathcal{C}_{j, j+1} E_{N, j+1}\left(t_{1}\right) d t_{1}
\end{aligned}
$$

shows that

$$
\left\|E_{N, j}(t)\right\|_{1} \leq \eta_{N, j}(t)+2 j\|V\|_{L^{\infty}} \int_{0}^{t}\left\|E_{N, j+1}(s)\right\|_{1} d s,
$$

since $\mathcal{S}_{n}(t)$ is an isometry of $\mathcal{E}_{n}$ while $\left\|\mathcal{C}_{n, n+1}\right\|_{\mathcal{L}\left(\mathcal{E}_{n+1}, \mathcal{E}_{n}\right)} \leq 2 j\|V\|_{L^{\infty}}$ (see step 4 in the proof of Theorem II.4), where

$$
\eta_{N, j}(t)=\left\|E_{N, j}(0)\right\|_{1}+\int_{0}^{t}\left\|\epsilon_{N, j}(s)\right\|_{1} d s .
$$

Iterating this inequality $m$ times leads to

$$
\begin{aligned}
\left\|E_{N, j}(t)\right\|_{1} & \leq \sum_{k=0}^{m}\left(\begin{array}{c}
j+k-1 \\
j-1
\end{array}\right)\left(2 j\|V\|_{L^{\infty}} t\right)^{k} \eta_{N, j+k}(t) \\
& +\left(\begin{array}{c}
j+m-1 \\
j-1
\end{array}\right)\left(2 j\|V\|_{L^{\infty}} t\right)^{m} \sup _{0 \leq s \leq t}\left\|E_{N, j+m+1}(s)\right\|_{1}
\end{aligned}
$$

Observing that

$$
\left\|\eta_{N, j}(t)\right\|_{1} \leq\left\|E_{N, j}(0)\right\|_{1}+2 j(j-1)\|V\|_{L^{\infty}} t\left(\frac{2}{N}+\|F(0)\|\right)
$$

and that

$$
\sup _{0 \leq s \leq t}\left\|E_{N, j+m+1}(s)\right\|_{1} \leq 2,
$$

the inequality (III.28) gives the announced convergence statement.

Before giving the proof of Proposition III.4, let us comment on step 4 above. The first proof of the Hartree approximation - Theorem II.4 — given by Spohn in [30] is based upon iterating Duhamel's formula (III.27). In this proof, one first establishes that

$$
\begin{array}{r}
D_{N: 1}(t) \rightarrow \mathcal{S}_{1}(t) D_{N: 1}(0) \\
+\sum_{k \geq 1}(-i)^{m} \int_{0<t_{m}<\ldots<t_{1}<t} \mathcal{S}_{1}\left(t-t_{1}\right) \mathcal{C}_{1,2} \\
\ldots \mathcal{C}_{m, m+1} \mathcal{S}_{m+1}\left(t_{m}\right) D_{N: m+1}(0) d t_{m} \ldots d t_{1}
\end{array}
$$

in $\mathcal{L}^{1}(\mathfrak{H})$ as $N \rightarrow+\infty$, by arguments similar to those used in step 4 . Finally, one recognizes the solution to Hartree's equation in the series on the right-hand 
side above. It may seem surprising that, in this type of proof, one works directly with explicit formulas giving the solutions to both hierarchies (BBGKY or infinite BBGKY) instead of working on the hierarchies themselves. This method of proof was used originally in the proof by Gallavotti [16] of the Boltzmann-Grad limit for the Lorentz gas with random distribution of scatterers, and later by Lanford [22] in his derivation of the Boltzmann equation from the dynamics of a hard sphere gas.

Proof of Proposition III.4. First, observe that

$$
\mathcal{R}_{j}(F)=\left(\sum_{k \neq l} V_{k, j+1} U_{l, j+1}\left(F_{j}^{-} \otimes F\right)\right)_{: j}-\left(\left(F_{j}^{-} \otimes F\right) \sum_{k \neq l} U_{l, j+1} V_{k, j+1}\right)_{: j}
$$

since

$$
U_{k, j+1} \text { commutes with } F^{\otimes j+1}
$$

and

$$
\Sigma_{j} \otimes I \text { commutes with } \sum_{\substack{1 \leq k, l \leq n \\ k \neq l}} V_{k, j+1} U_{l, j+1}
$$

(check this!) Hence, by symmetry, we have

$$
\left\|\mathcal{R}_{j}(F)\right\|_{1} \leq 2 j(j-1)\left\|\left(V_{j-1, j+1} U_{j, j+1} F_{j}^{-} \otimes F\right)_{: j}\right\|_{1} .
$$

Next, observe that

$$
\begin{aligned}
\left(V_{j-1, j+1} U_{j, j+1} F_{j}^{-} \otimes F\right)_{: j} & =\left(U_{j, j+1}\left(V_{j-1, j} F_{j}^{-}\right) \otimes F\right)_{: j} \\
& =\left(I^{\otimes j-1} \otimes F\right)\left(V_{j-1, j} F_{j}^{-}\right) .
\end{aligned}
$$

(Indeed, an easy computation shows that, if $A$ and $B$ are integral operators on $\mathfrak{H}=$ $L^{2}\left(\mathbf{R}^{D}\right)$ with integral kernels denoted respectively by $a \equiv a(x, y)$ and $b \equiv b(x, y)$, then

$$
\left(U_{12}(A \otimes B)\right)_{: 1}=B A,
$$

since

$$
U_{12}(A \otimes B) \text { has integral kernel } a\left(x_{2}, y_{1}\right) b\left(x_{1}, y_{2}\right) \text {, }
$$

so that

$$
\left(U_{12}(A \otimes B)\right)_{: 1} \text { has integral kernel } \int a\left(z, y_{1}\right) b\left(x_{1}, z\right) d z,
$$

which is precisely the integral kernel of $B A$ ).

Therefore,

$$
\begin{aligned}
\left\|\left(V_{j-1, j+1} U_{j, j+1} F_{j}^{-} \otimes F\right)_{: j}\right\| & =\left\|\left(I^{\otimes j-1} \otimes F\right)\left(V_{j-1, j} F_{j}^{-}\right)\right\|_{1} \\
& \leq\left\|I^{\otimes j-1} \otimes F\right\|\left\|V_{j-1, j} F_{j}^{-}\right\|_{1} \\
& \leq\|F\|\|V\|_{L^{\infty}}\left\|F_{j}^{-}\right\|_{1} .
\end{aligned}
$$


As can be seen from this proof, it is essential that the summation inside the commutator defining $\mathcal{R}_{j}(F)$ in (III.22) bears on $l \neq k$. Only this part of the interaction term can be crushed by the operator norm of the solution to the TDHF equation. This justifies a posteriori the decomposition of the interaction term as a leading order contribution

$$
\sum_{k=1}^{j}\left[V_{k, j+1}, F_{j+1}^{-}\right]_{: j}
$$

while the remaining part of the interaction, collected in $\mathcal{R}_{j}(F)$, is of the order of $\|F\|$, i.e. $O(1 / N)$ in the present case: see the remark just before Step 3 in the proof of Theorem III.3.

\section{Part IV. Extensions, open problems and further reading}

In these notes, both the Hartree and (time-dependent) Hartree-Fock have been established assuming that the pairwise interaction potential is bounded.

However, there are many interesting situations where this pairwise potential is unbounded:

- for systems of interacting electrons, the only physically relevant potential is Coulomb's potential $V(r)=C /|r|$ (in three space dimensions), which is obviously unbounded;

- for a "quasi-perfect" gas of bosons near zero temperature, the relevant meanfield PDE is the nonlinear Schrödinger equation with cubic nonlinearity (see [21], §30), i.e. Hartree's equation with interaction potential of the form $\delta\left(x_{1}-\right.$ $x_{2}$ ) (up to multiplication by some constant).

The first result for (a large class of) unbounded potentials - including in particular the case of Coulomb's potential — is the convergence of the finite BBGKY hierarchy (II.13) to the infinite BBGKY hierarchy (II.15), proved in [5]. The convergence result in [5] holds for any nonnegative potential in $C\left(\mathbf{R}^{D} \backslash\{0\}\right) \cap L_{l o c}^{2}\left(\mathbf{R}^{D}\right)$ vanishing at infinity.

The uniqueness for the infinite BBGKY hierarchy (II.15) in the case of the Coulomb potential is proved in [15] — see also [2]. The proof by Erdös and Yau follows the argument presented in part II of these lectures. The crucial part of their analysis lies in the choice of the sequence of Banach spaces $\mathcal{E}_{n}$ in the abstract uniqueness result of Proposition II.3: they choose to work with

$$
\mathcal{E}_{n}=\left\{T \in \mathcal{L}^{1}\left(\mathfrak{H}^{\otimes n}\right) \mid D^{\otimes n} T D^{\otimes n} \in \mathcal{L}^{1}\left(\mathfrak{H}^{\otimes n}\right\}\right.
$$

where $\mathfrak{H}=L^{2}\left(\mathbf{R}^{3}\right)$ and $D=\sqrt{I-\Delta}$ - i.e. the space of integral kernels with traceclass mixed derivatives. That $\mathcal{C}_{j, j+1}$ has norm at most $j$ as a bounded operator from $\mathcal{E}_{j+1}$ to $\mathcal{E}_{j}$ follows from a nice application of Hardy's inequality

$$
\left\|\frac{\phi}{|x|^{2}}\right\|_{L^{2}\left(\mathbf{R}^{3}\right)} \leq 2\|\nabla \phi\|_{L^{2}\left(\mathbf{R}^{3}\right)} .
$$


The next important part of their analysis consists in showing that $\left\|D_{n}\right\|_{\mathcal{E}_{n}}$ grows as $c^{n}$ for some $c>1$. In the case of a bounded potential, the analogous estimate is the fact that $\left\|D_{n}\right\|_{1} \leq 1$, which follows from the conservation of mass (i.e. of $\operatorname{trace}\left(D_{N}(t)\right)$ for all $t \geq 0$ and all $\left.N \geq 1\right)$ under the $N$-body Schrödinger dynamics. The analogous estimate in $\mathcal{E}_{n}$ is much trickier and follows from the conservation of energy under that same dynamics and an argument that compares powers of the free Hamiltonian

$$
\sum_{k=1}^{N}-\frac{1}{2} \Delta_{x_{k}}
$$

with powers of the interaction Hamiltonian

$$
\sum_{k=1}^{N}-\frac{1}{2} \Delta_{x_{k}}+\frac{1}{N} \sum_{1 \leq k<l \leq N} \frac{1}{\left|x_{k}-x_{l}\right|} .
$$

Again, this is based upon variants of Hardy's inequality above.

More recently, the proof of the TDHF approximation presented in part III above was extended to the case of Coulomb interactions, in [4]. The proof is based on the ideas in [3] and [15]. It requires more than the uniqueness estimate in [15], namely an estimate of the difference between both hierarchies (i.e. the BBGKY (II.13) and TDHF (III.21) hierarchies). This leads to additional technicalities that are beyond the scope of these lectures.

In the case of a delta potential in space dimension 1, the convergence of the BBGKY (II.13) to the infinite BBGKY hierarchy (II.15) is proved in [1]. This is a first step towards a derivation of the (cubic) nonlinear Schrödinger equation.

In the case of the classical mean-field limits, there is no derivation so far of the Vlasov-Poisson system from the classical $N$-body problem with Coulomb potential (even the definition of the dynamics in this case is not obvious).

Finally, one could think of proving the mean-field plus semi-classical limit, starting from the $N$-body Schrödinger equation (1.20) and with the Vlasov equation (1.1) as a target. There is a very interesting contribution by Narnhofer and Sewell [25] on this problem; however, it requires a real-analytic potential.

Acknowledgements. The material presented in these lectures is in places far from original, and I am indebted to several colleagues who taught me this subject. In particular, I learned Dobrushin's beautiful argument from Mario Pulvirenti, who gave a lucid presentation of mean-field limits in a lecture at Ecole Normale Superieure in 1997. I learned the idea of using Nishida's version of the Cauchy-Kovalevski theorem to handle the BBGKY hierarchy from a talk by Seiji Ukai at Sone's seminar in Kyoto in 1998; Ukai's elegant argument simplified part of Lanford's derivation of the Boltzmann equation originally phrased in terms of the perturbation series presented in part III. I also wish to thank my collaborators C. Bardos, A. Gottlieb and N. Mauser with whom I have worked on mean-field limits in the last few years. Our collaboration owes much to HyKE (EU financed network no. HPRN-CT-2002-00282). Also the four of us benefited from several discussions on the TDHF problem with C. Le Bris, X. Blanc, E. Cances and B. Ducomet: it is a pleasure to thank them all for their most generous help. Finally, I express my gratitude to the organizers of this conference for their kind invitation. 


\section{Part References}

[1] R. Adami, C. Bardos, F. Golse, A. Teta, Towards a rigorous derivation of the cubic nonlinear Schrödinger equation in dimension 1, preprint.

[2] C. Bardos, L. Erdös, F. Golse, N. Mauser, H.-T. Yau, Derivation of the Schrödinger-Poisson equation from the quantum $N$-body problem, C. R. Acad. Sci. Sér. I Math 334 (2002), 515-520.

[3] C. Bardos, F. Golse, A. Gottlieb, N. Mauser, Mean field dynamics of fermions and the time-dependent Hartree-Fock equation, to appear in J. de Math. Pures et Appl. 82 (2003).

[4] C. Bardos, F. Golse, A. Gottlieb, N. Mauser, Derivation of the Time-Dependent Hartree-Fock Equation with Coulomb Potential, in preparation.

[5] C. Bardos, F. Golse, N. Mauser, Weak coupling limit of the N-particle Schrödinger equation, Methods Appl. Anal. 7 (2000), no. 2, 275-293.

[6] A. Bove, G. DaPrato, G. Fano, An existence proof for the Hartree-Fock timedependent problem with bounded two-body interaction, Commun. Math. Phys. 37 (1974), 183-191.

[7] A. Bove, G. DaPrato, G. Fano, On the Hartree-Fock time-dependent problem, Comm. Math. Phys. 49 (1976), 25-33.

[8] W. Braun, K. Hepp, The Vlasov Dynamics and Its Fluctuations in the $1 / N$ Limit of Interacting Classical Particles; Commun. Math. Phys. 56 (1977), 101113.

[9] E. Caglioti, P.-L. Lions, C. Marchioro, M. Pulvirenti: A special class of flows for two-dimensional Euler equations: a statistical mechanics description, Commun. Math. Phys. 143 (1992), 501-525.

[10] E. Cancès, C. Le Bris, On the time-dependent Hartree-Fock equations coupled with a classical nuclear dynamics, Math. Models Methods Appl. Sci. 9 (1999), 963-990.

[11] I. Catto, Isabelle, C. Le Bris, P.-L. Lions, The mathematical theory of thermodynamic limits: Thomas-Fermi type models, Oxford Mathematical Monographs, The Clarendon Press, Oxford University Press, New York, (1998).

[12] J.M. Chadam, R.T. Glassey, Global existence of solutions to the Cauchy problem for time-dependent Hartree equations, J. Mathematical Phys. 16 (1975), 11221130 .

[13] L. Desvillettes, C. Villani, On the trend to global equilibrium for spatially inhomogeneous kinetic systems: the Boltzmann equation; preprint.

[14] R. Dobrushin, Vlasov equations; Funct. Anal. Appl. 13 (1979), 115-123. 
[15] L. Erdös, H.-T. Yau: Derivation of the nonlinear Schrödinger equation from a many body Coulomb system, Adv. Theor. Math. Phys. 5 (2001), 1169-1205.

[16] G. Gallavotti, Rigorous theory of the Boltzmann equation in the Lorentz gas, Nota int. no. 358, Istituto di Fisica, Università di Roma, (1972). Reprinted in Statistical Mechanics: a Short Treatise, pp. 48-55, Springer-Verlag BerlinHeidelberg (1999)

[17] M. Kiessling, Statistical mechanics of classical particles with logarithmic interactions; Commun. Pure Appl. Math. 46 (1993), 27-56.

[18] F. King, PhD Thesis, U. of California, Berkeley 1975.

[19] L. Landau, E. Lifshitz: Mécanique quantique; Editions Mir, Moscou 1967.

[20] L. Landau, E. Lifshitz: Théorie quantique relativiste, première partie; Editions Mir, Moscou 1972.

[21] L. Landau, E. Lifshitz: Physique statistique, deuxième partie; Editions Mir, Moscou 1990.

[22] O. Lanford: Time evolution of large classical systems, in "Dynamical systems, theory and applications" (Rencontres, Battelle Res. Inst., Seattle, Wash., 1974), pp. 1-111. Lecture Notes in Phys., Vol. 38, Springer, Berlin, 1975.

[23] C. Marchioro, M. Pulvirenti Mathematical Theory of Incompressible Nonviscous Fluids, Springer-Verlag (1994).

[24] V. Maslov: Equations of the self-consistent field; J. Soviet Math. 11 (1979), 123-195.

[25] H. Narnhofer, G.L. Sewell, Vlasov hydrodynamics of a quantum mechanical model, Comm. Math. Phys. 79 (1981), 9-24.

[26] H. Neunzert The Vlasov equation as a limit of Hamiltonian classical mechanical systems of interacting particles; Trans. Fluid Dynamics 18 (1977), 663-678.

[27] L. Nirenberg An abstract form of the nonlinear Cauchy-Kowalewski theorem; J. Differential Geometry 6 (1972), 561-576.

[28] L. Onsager Statistical hydrodynamics, Supplemento al Nuovo Cimento 6 (1949), 279-287.

[29] T. Nishida A note on a theorem of Nirenberg; J. Differential Geometry 12 (1977), 629-633.

[30] H. Spohn, Kinetic Equations from Hamiltonian Dynamics: Markovian Limits, Rev. Modern Phys. 52 (1980), 569-615. 
Institut Universitaire De France

\& LABORATOIRE JACQUES-LOUIS LIONS

Bô̂te COURRIER 187, 4 PLACE Jussieu

F75252 PARIS CEDEX 05

golse@math.jussieu.fr

www.dma.ens.fr $/ \sim$ golse 